\title{
Assessment of groundwater quality from Bankura I and II Blocks, Bankura District, West Bengal, India
}

\author{
S. K. Nag $^{1} \cdot$ Shreya Das ${ }^{1}$
}

Received: 17 March 2015/Accepted: 11 January 2017/Published online: 13 February 2017

(C) The Author(s) 2017. This article is published with open access at Springerlink.com

\begin{abstract}
Hydrochemical evaluation of groundwater has been conducted in Bankura I and II Blocks to analyze and determining groundwater quality in the area. Thirty-six groundwater samples were analyzed for their physical and chemical properties using standard laboratory methods. The constituents have the following ranges in the water: $\mathrm{pH}$ 6.4-8.6, electrical conductivity $80-1900 \mu \mathrm{S} / \mathrm{cm}$, total hardness $30-730 \mathrm{mg} / \mathrm{l}$, TDS $48-1001 \mathrm{mg} / \mathrm{l}, \mathrm{Ca}^{2+} 4.2-$ $222.6 \mathrm{mg} / \mathrm{l}, \quad \mathrm{Na}^{+} \quad 2.33-103.33 \mathrm{mg} / \mathrm{l}, \quad \mathrm{Mg}^{2+} \quad 1.56-$ $115.36 \mathrm{mg} / \mathrm{l}, \mathrm{K}^{+} 0.67-14 \mathrm{mg} / \mathrm{l}$ and $\mathrm{Fe}$ BDL-2.53 mg/l, $\mathrm{HCO}_{3}^{-} 48.8-1000.4 \mathrm{mg} / \mathrm{l}, \mathrm{Cl}^{-} 5.6-459.86 \mathrm{mg} / \mathrm{l}$ and $\mathrm{SO}_{4}^{=}$ BDL-99.03 mg/l. Results also show that bicarbonate ions $\left(\mathrm{HCO}_{3}^{-}\right)$dominate the other anions $\left(\mathrm{Cl}^{-}\right.$and $\left.\mathrm{SO}_{4}^{2-}\right)$. Sodium adsorption ratio (SAR), soluble sodium percentage (SSP), residual sodium carbonate (RSC), magnesium adsorption ratio (MAR), total hardness (TH), and permeability index (PI) were calculated as derived parameters, to investigate the ionic toxicity. Concerned chemical parameters when plotted in the U.S. Salinity diagram indicate that waters are of $\mathrm{C}_{1}-\mathrm{S}_{1}, \mathrm{C}_{2}-\mathrm{S}_{1}$ and $\mathrm{C}_{3}-\mathrm{S}_{1}$ types, i.e., low salinity and low sodium which is good for irrigation. The values of Sodium Adsorption Ratio indicate that the groundwater of the area falls under the category of low sodium hazard. So, there is neither salinity nor toxicity problem of irrigation water, and hence the ground water can safely be used for long-term irrigation. The chemical parameters when plotted in Piper's trilinear diagram are found to concentrate in the central and west central part of the diamond-shaped field. Based on the analytical results, groundwater in the area is found to be generally fresh and
\end{abstract}

\footnotetext{
S. K. Nag

nag_sk@yahoo.com

Jadavpur University, Kolkata, West Bengal, India
}

hard to very hard. The abundance of the major ions is as follows: $\mathrm{HCO}_{3}>\mathrm{Cl}>\mathrm{SO}_{4}$ and $\mathrm{Ca}>\mathrm{Na}>\mathrm{Mg}>\mathrm{K}>$ $\mathrm{Fe}$. Results also show that bicarbonate ions $\left(\mathrm{HCO}_{3}^{-}\right)$ dominate the other anions $\left(\mathrm{Cl}^{-}\right.$and $\left.\mathrm{SO}_{4}^{2-}\right)$. According to Gibbs diagrams samples fall in the rock dominance field and the chemical quality of groundwater is related to the lithology of the area. The alkaline earth elements $(\mathrm{Ca}$ and $\mathrm{Mg}$ ) occur in greater abundance than alkaline elements ( $\mathrm{Na}$ and $\mathrm{K}$ ). A comparative study of our analytical results with the WHO standards of drinking water indicate that the present waters are also good for drinking purposes.

Keywords Hydrochemistry · Water quality · Domestic and irrigation suitability $\cdot$ Spatial distribution $\cdot$ Bankura

\section{Introduction}

Safe portable water is absolutely essential for healthy living. Groundwater is renewable natural resources and is one of the pure sources of water because it is bacteriologically free and contains more health required nutrients in the right proportion than surface water. For this reason it is safe to use as drinking water sources and consumption purposes. It can be used for both domestic and industrial purposes; it is a continuous source of water that is inexhaustible the, reliable and utilizable. It is estimated that approximately one-third of the world's population use groundwater for drinking purposes. Water shortages have become an increasingly serious problem in India, especially in the arid and semi-arid regions of the country due to vagaries of monsoon and scarcity of surface water. In India, groundwater constitutes about $53 \%$ of the total irrigation potential and about $50 \%$ of the total irrigated area is dependent on groundwater irrigation (Central Water Commission 2006).

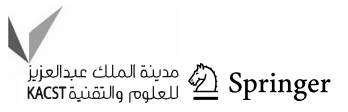


The quality of groundwater is the resultant of all the processes and reaction that act on the water from the moment it condenses in the atmosphere to the time it is discharged by a well. Therefore, determination of groundwater quality is important to observe the suitability of water for a particular use. The problems of ground water quality are more acute in areas that are densely populated and thickly industrialized and have shallow groundwater tube wells. In developing world, $80 \%$ of diseases are directly related to poor drinking water and unsanitary conditions (UNESCO 2007). Geochemical studies of groundwater provide a better understanding of possible changes in quality as development progress. Suitability of groundwater for domestic and irrigation purposes is determined by its groundwater geochemistry. Groundwater quality data give important clues to the geologic history of rocks and indications of groundwater recharge, movement and storage (Walton 1970). Groundwater quality depends on number of factors, such as general geology, degree of chemical weathering of prevailing lithology, quality of recharge water and inputs from sources other than water-rock interaction (Hussein 2004; Schuh et al. 1997; Freeze and Cherry 1979; Domenico 1972). Demarcation of groundwater zones on the basis of quality was attempted by Subba Rao et al. (2002) in Guntur of AP, India. Lithological influence and dominance of anthropogenic factors on groundwater chemistry in Salem district, TN, India was attempted by Srinivasamoorthy et al. (2008). Identification of geochemical facies and demarcation of locations unfit for human consumption was attempted by Mohan et al. (2000) in UP state of India. Nitrate contamination is strongly related to land use pattern has been attempted by Rajmohan et al. (2007). Similar studies in different parts of the globe have also been attempted by Bathrellos et al. (2008); Ahmed et al. (2002) and Stites and Kraft (2001). India has been facing the problem of deteriorating groundwater quality due to rapid urbanization and its ever increasing population at an exponential rate (Brindha et al. 2011; Brindha and Elango 2010, 2011; Ramesh and Elango 2005). Temporal changes in the origin and constitution of the recharged water, hydrological and human factors frequently cause periodic changes in groundwater quality (Aghazadeh and Mogaddam 2010; Milovanovic 2007; Sreedevi 2004). Many research publications have come out on evaluation for domestic and industrial activities and related groundwater quality monitoring (Vasanthavigar et al. 2010; Pritchard et al. 2008; Al-Futaisi et al. 2007; Jalali 2007; Mukherjee and Das 2007; Rivers et al. 1996).

Earlier, the crystalline rocks or so-called hard rocks received less attention from groundwater point of view due to their low permeability and also difficulties in drilling (Singhal and Gupta 2010). But during the last few decades, owing to the needs for safe drinking water for vast population, these crystalline rocks are being investigated in detail for groundwater development (Ahmed 2007; Lloyd 1999; Wright and Burgess 1992). Fractures often serve as major conduits for groundwater movement. The rock types commonly encountered in the study area are granite or granite gneisses overlain by a variable thickness of weathered material. The weathered material is a regolith produced by the in situ weathering of the basement rock (Acworth 1987). Similar studies based on groundwater quality and hydrogeochemistry in different blocks of Bankura and Purulia districts of West Bengal have been taken up by many researchers (Nag and Ray 2015; Nag 2014; Nag and Ghosh 2013).

In view of the above discussions, it is important to ascertain the groundwater quality of the area for domestic and irrigational purposes. The objective of this paper is to use hydrochemical method to assess the suitability of groundwater in the area for domestic as well as irrigation purposes.

\section{Study area}

In West Bengal, the Bankura district is a semi-arid and drought prone area as the Tropic of Cancer passes through it. The present study area includes the Bankura Block I and Block II (Fig. 1) between $23^{\circ} 09^{\prime} 24^{\prime \prime}-23^{\circ} 22^{\prime} 51^{\prime \prime}$ North latitude and $86^{\circ} 53^{\prime} 51^{\prime \prime}-87^{\circ} 14^{\prime} 19^{\prime \prime}$ East longitude covering an area of 411.42 square kilometers (Block $\mathrm{I}=189.18$ square kilometers and Block $\mathrm{II}=222.24$ square kilometers). The area is predominantly an undulated Precambrian hilly terrain with sporadic soil cover. It is a thinly populated district and the population is moderately concentrated in Block I and Block II because the Bankura town is the district headquarters.

The economy of the area is based on agriculture and the agriculture is dependent partly on groundwater because the rivers in this area are perennial and lacks any canal facility. Thus, the poor people of Bankura district are largely dependent on groundwater for both domestic as well as agricultural purposes.

The study area is characterized by gently to moderately rolling plain with lateritic uplands, valley cuts and terraced banks. Regionally, the area constitutes the extreme eastern fringe of the Ranchi Plateau and further east gradually merges with the depositional fluvial terraces of Dwarakeswar-Gandheswari Rivers. The regional south-easterly slope is exemplified by the Dwarakeswar River which flows from northwest to southeast. The overall drainage pattern of the area is parallel to sub-parallel and is mainly controlled by geological structural elements. The country rocks are Chotanagpur granite gneiss with enclaves of meta-sedimentaries. Major portion of the block is 


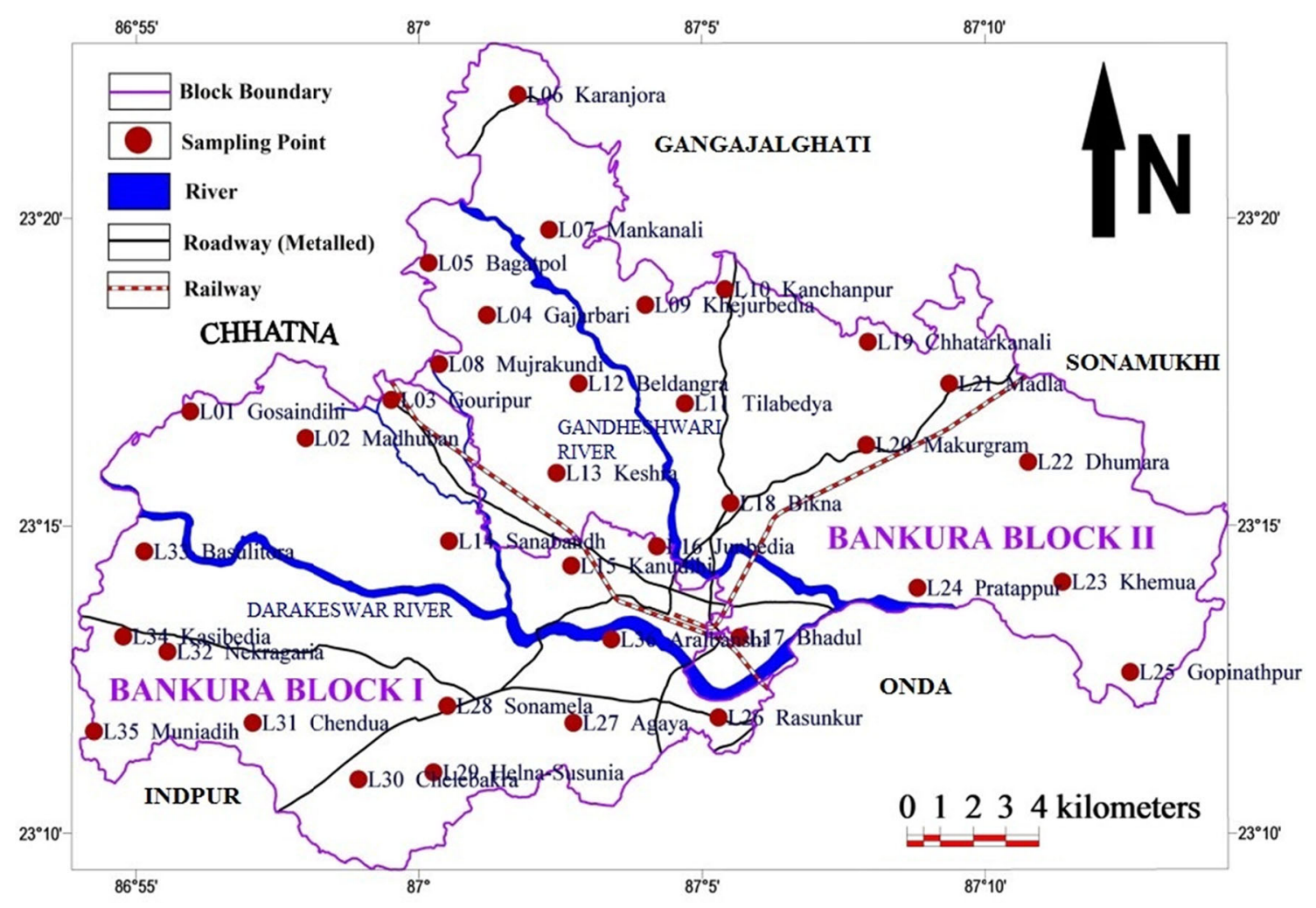

Fig. 1 Map of the study area showing sample locations

characterized by the presence of skeletal soil, at places lateritic also, except the valley fills (Nag and Ghosh 2013) (Fig. 2).

Hydrogeologically the weathered overburden is characterized by high porosity and contains a significant amount of water, but, due to its relatively high clay content, it has a low permeability. The basement rock, on the other hand, is relatively fresh frequently fractured and thereby producing high permeability (Krishnamurthy et al. 2007; Dewandel et al. 2006).

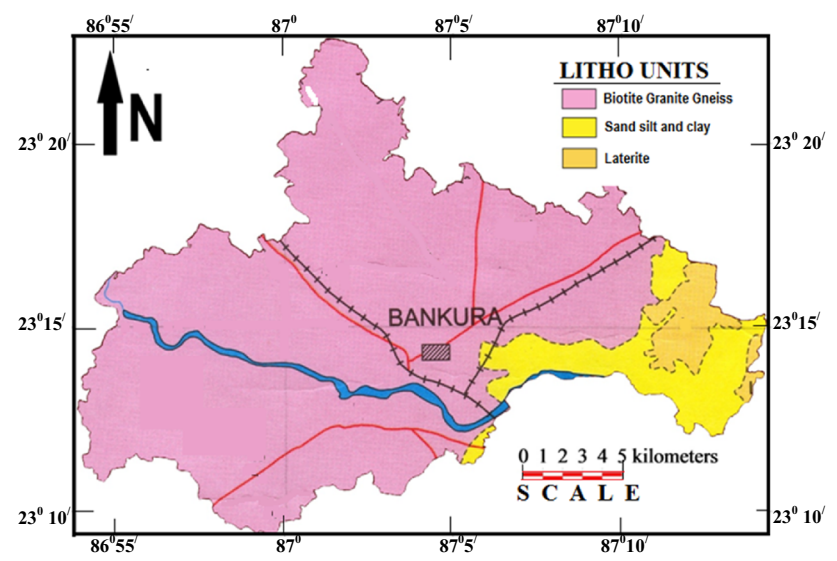

Fig. 2 Lithological units of the study area

\section{Data used and methodology}

To access the quality of the groundwater of the present area 36 bore wells were located covering the whole study area more or less uniformly (Fig. 1). Ground water samples were collected from all 36 locations in the field in both premonsoon (April 2012) and post-monsoon (October 2012) time. The bore wells were pumped for some time before collecting water samples so as to eliminate the residual water in the casing tube of the well and to obtain fresh water directly from the aquifer. Dry, clean and sterilized plastic bottles were used to get fresh aquifer water for sampling. Water samples were collected in air tight preconditioned high-density polythene bottles of $500 \mathrm{ml}$ capacity. All bottles were thoroughly washed and rinsed with water before collecting ground water samples. The polythene bottles were completely filled without any air bubble before sealing the cap. The locations from where water samples have been collected are marked on the study area map by hand held portable Global Positioning System (GPS) device. GPS provides us with longitude, latitude and elevation with respect to mean sea level (MSL) of the location. All particulars regarding water sample were noted in the field itself, immediately after sampling, and tagged to the sample bottle. Special treatments were given for preservation, fixation and handling of water samples before analysis. Otherwise, the quality of water may change and 
many of heavy metals ions normally present in small quantities in natural water may not remain in water till the sample is analyzed. Seal of the bottles were checked before storage. High temperature and direct sunlight were avoided in the storage room. The samples were free of sediments and acidified to about a $\mathrm{pH}$ of 3.5 with glacial acetic acid at the time of collection. A little formaldehyde $(0.2 \mathrm{~m} /$ $100 \mathrm{ml}$ ) is added to retard mould growth. Seals of the bottles are tightened before storage. High temperature was avoided in the storage room.

Monitoring was done during pre- (April, 2012) and postmonsoon (November, 2012). Only high quality pure chemicals and double-distilled water were used for preparing solutions for analysis. In the present study, base map showing locations of investigating points has been prepared using Survey of India (SoI) Topo sheets 73 I/15, 73 I/16, 73 M/3 and $73 \mathrm{M} / 4$ and satellite imagery (IRS-IB, LISS-II). The GIS and image processing software TNT Mips 2012 has been used to prepare the study area maps. The maps available have been scanned and imported into TNT Mips 2012 and the locations of the sampling points have been imported through point import function. Groundwater samples were analyzed in the laboratory for the major ions chemistry employing standard method (APHA 1998). Parameters like pH, Electrical conductivity (EC), Total Dissolved solids (TDS) were determined at the site using pHTestr 2 and ECTestr+ by Eutech Instruments and DIST 3 by Hanna Instruments, respectively. Calcium $\left(\mathrm{Ca}^{2+}\right)$ and magnesium $\left(\mathrm{Mg}^{2+}\right)$ were determined titrimetrically using standard EDTA. Chloride $\left(\mathrm{Cl}^{-}\right)$by standard $\mathrm{AgNO}_{3}$ titration, bicarbonate $\left(\mathrm{HCO}_{3}^{-}\right)$by titration with $\mathrm{HCl}$, sodium $\left(\mathrm{Na}^{+}\right)$and potassium $\left(\mathrm{K}^{+}\right)$by flame photometry. The analytical precision for ions was determined by the ionic balances calculated as $100 \times$ (cations - anions)/(cations + anions), which is generally within $\pm 5 \%$ (Srinivasamoorthy et al. 2011).The results of the physico-chemical parameters of the 36 samples are shown in Table 1 (both pre- and post-monsoon).

The parameters such as sodium adsorption ratio (SAR), soluble sodium percentage (SSP), residual sodium carbonate (RSC), magnesium adsorption ratio (MAR), total hardness $(\mathrm{TH})$, and permeability index (PI) were calculated to evaluate the suitability of the water quality for agricultural purposes and are shown in Table 2. Further, the results of the analyses were interpreted using different graphical representations.

\section{Results and discussion}

Access to safe drinking water remains an urgent necessity, as $30 \%$ of urban and $90 \%$ of rural households still depend completely on untreated surface or groundwater (Kumar et al. 2005). While access to drinking water in India has increased over the past decade, the tremendous adverse impact of unsafe water on health continues (WHO/UNICEF 2004). It is now generally recognized that the quality of groundwater is just as important as its quantity.

\section{Major ion chemistry and spatial distribution}

The $\mathrm{pH}$ values of the groundwater varies from 6.4 to 7.6 (in pre-monsoon) with an average of 7.17, and 7.1-8.6 (in post-monsoon) with an average of 7.83 , which indicates that water is slightly acidic in nature. The electrical conductivity values were found between 100 and 1900 (in premonsoon) with an average of $705 \mu \mathrm{s} / \mathrm{cm}$ and $80-430$ with an average of $291 \mu \mathrm{s} / \mathrm{cm}$ (in post-monsoon) at $25{ }^{\circ} \mathrm{C}$ in the study area. Total Dissolved Solids (TDS) ranged from 51 to $949 \mathrm{mg} / \mathrm{l}$ in pre-monsoon and $48-1001 \mathrm{mg} / \mathrm{l}$ in post-monsoon. The average concentration of total dissolved solids (TDS) ranged from 343.13 (pre-monsoon) to 353.08 (postmonsoon) $\mathrm{mg} / \mathrm{l}$ in the study area. Normally TDS in water may originate from natural sources and sewage discharges. The Total hardness in water is derived from the solution of carbon dioxide released by bacterial action in the soil, in percolating rain water. Low $\mathrm{pH}$ conditions develop and lead to the dissolution of insoluble carbonates in the soil and in limestone formations to convert them into soluble bicarbonates. Impurities in limestone, such as sulfates, chlorides and silicates, become exposed to the solvent action of water as the carbonates are dissolved so that they also pass into solution. The general acceptance level of hardness is $300 \mathrm{mg} / \mathrm{l}$, although WHO has set an allowable limit of $600 \mathrm{mg} / \mathrm{l}$. Total Hardness ranged from 48 to $624 \mathrm{mg} / \mathrm{l}$ with an average value of $250.67 \mathrm{mg} / \mathrm{l}$ in premonsoon and $30-730 \mathrm{mg} / \mathrm{l}$ with an average value of $277.5 \mathrm{mg} / \mathrm{l}$ in post-monsoon. Total alkalinity (TA) ranged from 40 to $390 \mathrm{mg} / \mathrm{l}$ in pre-monsoon and $70-820 \mathrm{mg} / \mathrm{l}$ in post-monsoon. The average concentration of total alkalinity (TA) ranged from 185.83 (pre-monsoon) to 397.22 (post-monsoon) $\mathrm{mg} / \mathrm{l}$ in the study area.

Iron is an essential element in human (Moore 1973). Although iron has little concern as a health hazard, it is still considered as a nuisance in excessive quantities (Dart 1974). It causes staining of clothes and utensils. It is also not suitable for processing of food, beverages, dyeing, bleaching, etc. The concentration limits of iron in drinking water ranges between $0.3 \mathrm{mg} / \mathrm{l}$ (maximum acceptable) and $1.0 \mathrm{mg} / \mathrm{l}$ (maximum allowable) (Sharma and Chawla 1977). Fe ranges from 0 to $3.577 \mathrm{mg} / \mathrm{l}$ in pre-monsoon and $0-2.53 \mathrm{mg} / \mathrm{l}$ in post-monsoon. High iron concentration affects the taste. It has adverse effects on domestic uses and promotes growth of iron bacteria. 
Table 1 Physicochemical parameters of groundwater samples in pre- and post-monsoon season 2012

\begin{tabular}{|c|c|c|c|c|c|c|c|c|c|c|c|c|c|c|}
\hline \multirow[t]{2}{*}{ Location No. } & \multicolumn{2}{|l|}{$\mathrm{pH}$} & \multicolumn{2}{|l|}{ TDS } & \multicolumn{2}{|l|}{$\mathrm{EC}$} & \multicolumn{2}{|l|}{ TA } & \multicolumn{2}{|l|}{$\mathrm{TH}$} & \multicolumn{2}{|l|}{$\mathrm{Ca}^{2+}$} & \multicolumn{2}{|l|}{$\mathrm{Mg}^{2+}$} \\
\hline & Pre & Post & Pre & Post & Pre & Post & Pre & Post & Pre & Post & Pre & Post & Pre & Post \\
\hline L01 & 7.40 & 8.00 & 217.00 & 243.00 & 500 & 240 & 120.00 & 280.00 & 176.00 & 180.00 & 47.04 & 42.00 & 14.25 & 18.30 \\
\hline L02 & 7.10 & 8.10 & 334.00 & 479.00 & 800 & 300 & 200.00 & 380.00 & 256.00 & 310.00 & 47.04 & 67.20 & 33.77 & 34.65 \\
\hline L03 & 7.30 & 8.20 & 244.00 & 283.00 & 600 & 270 & 260.00 & 90.00 & 256.00 & 430.00 & 67.20 & 54.60 & 21.47 & 71.61 \\
\hline L04 & 7.40 & 7.10 & 51.00 & 48.00 & 100 & 80 & 60.00 & 400.00 & 64.00 & 240.00 & 20.16 & 8.40 & 3.32 & 53.44 \\
\hline L05 & 7.60 & 7.80 & 210.00 & 198.00 & 500 & 230 & 180.00 & 400.00 & 144.00 & 160.00 & 20.16 & 42.00 & 22.84 & 13.42 \\
\hline L06 & 7.10 & 7.50 & 644.00 & 630.00 & 1400 & 340 & 280.00 & 550.00 & 496.00 & 560.00 & 40.32 & 147.00 & 96.43 & 46.97 \\
\hline L07 & 7.40 & 7.80 & 359.00 & 244.00 & 900 & 280 & 280.00 & 460.00 & 256.00 & 130.00 & 20.16 & 21.00 & 50.17 & 18.91 \\
\hline L08 & 7.10 & 8.00 & 122.00 & 157.00 & 300 & 200 & 120.00 & 310.00 & 128.00 & 170.00 & 40.32 & 29.40 & 6.64 & 23.55 \\
\hline L09 & 7.60 & 7.80 & 406.00 & 457.00 & 900 & 350 & 220.00 & 410.00 & 272.00 & 300.00 & 47.04 & 63.00 & 37.67 & 34.77 \\
\hline L10 & 6.90 & 7.30 & 279.00 & 283.00 & 600 & 290 & 100.00 & 200.00 & 224.00 & 220.00 & 13.44 & 54.60 & 46.46 & 20.37 \\
\hline L11 & 7.10 & 7.40 & 416.00 & 445.00 & 900 & 350 & 180.00 & 380.00 & 320.00 & 350.00 & 73.92 & 84.00 & 32.99 & 34.16 \\
\hline L12 & 7.10 & 7.50 & 440.00 & 465.00 & 1000 & 360 & 200.00 & 520.00 & 256.00 & 360.00 & 26.88 & 96.60 & 46.07 & 28.91 \\
\hline L13 & 7.10 & 8.20 & 108.00 & 164.00 & 300 & 190 & 80.00 & 220.00 & 128.00 & 140.00 & 33.60 & 25.20 & 10.74 & 18.79 \\
\hline L14 & 7.40 & 8.60 & 247.00 & 317.00 & 600 & 300 & 180.00 & 420.00 & 192.00 & 260.00 & 47.04 & 37.80 & 18.15 & 40.38 \\
\hline L15 & 7.20 & 8.20 & 590.00 & 529.00 & 1300 & 370 & 300.00 & 610.00 & 480.00 & 350.00 & 40.32 & 92.40 & 92.52 & 29.04 \\
\hline L16 & 7.40 & 7.80 & 533.00 & 295.00 & 1000 & 310 & 200.00 & 580.00 & 320.00 & 240.00 & 47.04 & 50.40 & 49.39 & 27.82 \\
\hline L17 & 7.40 & 7.80 & 351.00 & 397.00 & 800 & 330 & 200.00 & 500.00 & 224.00 & 310.00 & 26.88 & 67.20 & 38.26 & 34.65 \\
\hline L18 & 7.40 & 7.80 & 142.00 & 150.00 & 300 & 210 & 80.00 & 300.00 & 176.00 & 130.00 & 53.76 & 29.40 & 10.15 & 13.79 \\
\hline L19 & 6.60 & 7.60 & 140.00 & 157.00 & 300 & 210 & 160.00 & 220.00 & 128.00 & 100.00 & 40.32 & 29.40 & 6.64 & 6.47 \\
\hline L20 & 6.60 & 7.10 & 383.00 & 373.00 & 800 & 360 & 200.00 & 350.00 & 240.00 & 260.00 & 80.64 & 75.60 & 9.37 & 17.32 \\
\hline L21 & 6.90 & 7.40 & 760.00 & 589.00 & 1300 & 400 & 390.00 & 750.00 & 624.00 & 460.00 & 60.48 & 105.00 & 115.36 & 48.19 \\
\hline L22 & 6.90 & 7.40 & 398.00 & 409.00 & 800 & 370 & 260.00 & 700.00 & 224.00 & 270.00 & 33.60 & 42.00 & 34.16 & 40.26 \\
\hline L23 & 6.40 & 7.20 & 105.00 & 103.00 & 200 & 130 & 80.00 & 190.00 & 80.00 & 120.00 & 20.16 & 16.80 & 7.22 & 19.03 \\
\hline L24 & 7.10 & 7.80 & 696.00 & 1001.00 & 1300 & 430 & 200.00 & 440.00 & 432.00 & 730.00 & 100.80 & 205.80 & 43.92 & 52.58 \\
\hline L25 & 6.60 & 7.10 & 86.00 & 53.00 & 200 & (ס) & 40.00 & 70.00 & 48.00 & 50.00 & 6.72 & 8.40 & 7.61 & 7.08 \\
\hline L26 & 7.40 & 7.80 & 402.00 & 380.00 & 900 & 350 & 360.00 & 820.00 & 224.00 & 270.00 & 26.88 & 50.40 & 38.26 & 35.14 \\
\hline L27 & 7.10 & 8.40 & 308.00 & 322.00 & 600 & 330 & 180.00 & 430.00 & 224.00 & 240.00 & 47.04 & 63.00 & 25.96 & 20.13 \\
\hline L28 & 7.50 & 8.40 & 416.00 & 434.00 & 1000 & 370 & 180.00 & 470.00 & 192.00 & 330.00 & 26.88 & 88.20 & 30.45 & 26.72 \\
\hline L29 & 7.10 & 8.20 & 949.00 & 932.00 & 1900 & 400 & 120.00 & 240.00 & 544.00 & 680.00 & 215.04 & 222.60 & 1.56 & 30.13 \\
\hline L30 & 6.70 & 7.80 & 660.00 & 684.00 & 1500 & 410 & 200.00 & 350.00 & 448.00 & 440.00 & 127.68 & 130.20 & 31.43 & 27.94 \\
\hline L31 & 7.60 & 8.50 & 93.00 & 84.00 & 200 & 100 & 140.00 & 80.00 & 112.00 & 30.00 & 33.60 & 4.20 & 6.83 & 4.76 \\
\hline L32 & 7.60 & 8.20 & 244.00 & 327.00 & 600 & 320 & 240.00 & 530.00 & 208.00 & 220.00 & 47.04 & 46.20 & 22.06 & 25.50 \\
\hline L33 & 7.20 & 8.20 & 258.00 & 258.00 & 600 & 310 & 120.00 & 310.00 & 208.00 & 240.00 & 60.48 & 63.00 & 13.86 & 20.13 \\
\hline L34 & 7.10 & 7.80 & 302.00 & 322.00 & 800 & 330 & 200.00 & 430.00 & 256.00 & 320.00 & 53.76 & 75.60 & 29.67 & 31.96 \\
\hline L35 & 7.40 & 8.00 & 227.00 & 228.00 & 600 & 280 & 180.00 & 490.00 & 224.00 & 200.00 & 47.04 & 54.60 & 25.96 & 15.49 \\
\hline L36 & 7.30 & 8.00 & 233.00 & 271.00 & 500 & 300 & 200.00 & 420.00 & 240.00 & 190.00 & 26.88 & 50.40 & 42.16 & 15.62 \\
\hline Min. & 6.40 & 7.10 & 51.00 & 48.00 & 100 & 80 & 40.00 & 70.00 & 48.00 & 30.00 & 6.72 & 4.2 & 1.56 & 4.76 \\
\hline Max. & 7.60 & 8.60 & 949.00 & 1001.00 & 1900 & 430 & 390.00 & 820.00 & 624.00 & 730.00 & 215.04 & 222.6 & 115.36 & 71.61 \\
\hline Mean & 7.17 & 7.83 & 343.14 & 353.08 & 705 & 291 & 185.83 & 397.22 & 250.67 & 277.50 & 49.09 & 65.1 & 31.21 & 28.00 \\
\hline Median & 7.15 & 7.80 & 305.00 & 319.50 & 700 & 310 & 190.00 & 405.00 & 224.00 & 250.00 & 43.68 & 54.6 & 27.81 & 27.27 \\
\hline Std.Dev. & 0.31 & 0.41 & 210.09 & 217.56 & 0.410 & 91 & 78.93 & 175.51 & 133.87 & 155.59 & 37.25 & 48.85 & 26.01 & 14.44 \\
\hline \multirow[t]{2}{*}{ Location No. } & \multicolumn{2}{|l|}{$\mathrm{Na}^{+}$} & \multicolumn{2}{|c|}{$\mathrm{K}^{+}$} & \multicolumn{2}{|l|}{$\mathrm{Fe}^{2+}$} & \multicolumn{2}{|l|}{$\mathrm{CO}_{3}^{2-}$} & \multicolumn{2}{|l|}{$\mathrm{HCO}_{3}^{-}$} & \multicolumn{2}{|l|}{$\mathrm{Cl}^{-}$} & \multicolumn{2}{|l|}{$\mathrm{SO}_{4}^{2-}$} \\
\hline & Pre & Pos & 11 & Post & Pre & Post & Pre & Post & Pre & Post & Pre & Post & Pre & Post \\
\hline Lut & 30.60 & & .00 & 5.00 & 0.138 & 0.03 & 24.00 & 0.00 & 97.60 & 341.60 & 39.99 & 69.98 & 19.23 & 19.01 \\
\hline L02 & 46.00 & & 0.00 & 1.40 & 0.029 & 0.03 & 48.00 & 0.00 & 146.40 & 463.60 & 54.98 & 159.95 & 14.72 & 24.69 \\
\hline L03 & 36.00 & & 2.20 & 1.20 & 0.023 & 0.42 & 48.00 & 0.00 & 219.60 & 109.80 & 59.98 & 29.99 & 30.05 & 2.04 \\
\hline
\end{tabular}


Table 1 continued

\begin{tabular}{|c|c|c|c|c|c|c|c|c|c|c|c|c|c|c|}
\hline \multirow[t]{2}{*}{ Location No. } & \multicolumn{2}{|l|}{$\mathrm{Na}^{+}$} & \multicolumn{2}{|l|}{$\mathrm{K}^{+}$} & \multicolumn{2}{|l|}{$\mathrm{Fe}^{2+}$} & \multicolumn{2}{|l|}{$\mathrm{CO}_{3}^{2-}$} & \multicolumn{2}{|l|}{$\mathrm{HCO}_{3}^{-}$} & \multicolumn{2}{|l|}{$\mathrm{Cl}^{-}$} & \multicolumn{2}{|l|}{$\mathrm{SO}_{4}^{2-}$} \\
\hline & Pre & Post & Pre & Post & Pre & Post & Pre & Post & Pre & Post & Pre & Post & Pre & Post \\
\hline L04 & 7.80 & 5.80 & 2.20 & 1.00 & 0.006 & 0.02 & 0.00 & 0.00 & 73.20 & 488.00 & 15.00 & 19.99 & 1.13 & 1.42 \\
\hline L05 & 30.40 & 27.20 & 5.80 & 2.80 & 0.081 & 0.15 & 24.00 & 144.00 & 170.80 & 195.20 & 19.99 & 29.99 & 7.01 & 0.62 \\
\hline L06 & 54.00 & 46.00 & 3.20 & 1.20 & 0.000 & 0.02 & 24.00 & 0.00 & 292.80 & 671.00 & 114.96 & 214.93 & 33.08 & 34.20 \\
\hline L07 & 60.00 & 70.00 & 4.40 & 2.00 & 0.035 & 0.06 & 48.00 & 24.00 & 244.00 & 512.40 & 39.99 & 24.99 & 14.03 & 0.71 \\
\hline L08 & 17.80 & 14.40 & 2.40 & 1.00 & 0.006 & 0.10 & 0.00 & 12.00 & 146.40 & 353.80 & 19.99 & 29.99 & 4.68 & 3.73 \\
\hline L09 & 37.20 & 37.60 & 10.20 & 14.00 & 0.178 & 2.53 & 48.00 & 0.00 & 170.80 & 500.20 & 69.98 & 139.96 & 23.04 & 9.59 \\
\hline L10 & 22.20 & 17.40 & 5.80 & 2.60 & 0.023 & 0.26 & 0.00 & 0.00 & 122.00 & 244.00 & 69.98 & 109.97 & 6.67 & 0.00 \\
\hline L11 & 54.00 & 52.00 & 6.60 & 3.00 & 0.075 & 0.04 & 48.00 & 0.00 & 122.00 & 463.60 & 79.98 & 154.95 & 38.10 & 41.12 \\
\hline L12 & 72.00 & 60.00 & 3.60 & 1.40 & 0.040 & 0.02 & 24.00 & 0.00 & 195.20 & 292.80 & 74.98 & 139.96 & 39.49 & 46.10 \\
\hline L13 & 24.60 & 19.80 & 2.80 & 1.20 & 0.012 & 0.04 & 0.00 & 0.00 & 97.60 & 268.40 & 39.99 & 49.98 & 2.17 & 1.07 \\
\hline L14 & 32.20 & 37.40 & 2.20 & 1.00 & .098 & 0.26 & 0.00 & 12.00 & 219.60 & 488.00 & 44.99 & 64.98 & 5.37 & 1.87 \\
\hline L15 & 0.00 & 93.33 & 5.40 & 3.67 & 0.023 & 0.34 & 72.00 & 60.00 & 219.60 & 622.20 & 84.97 & 119.96 & 57.94 & 52.85 \\
\hline L16 & 102.00 & 45.00 & 9.60 & 4.00 & 0.046 & 0.00 & 24.00 & 0.00 & 195.20 & 707.60 & 94.97 & 29.99 & 43.56 & 31.71 \\
\hline L17 & 46.00 & 40.33 & 2.80 & 2.00 & 0.012 & 0.05 & 72.00 & 0.00 & 97.60 & 610.00 & 74.98 & 124.96 & 10.05 & 11.99 \\
\hline L18 & 26.60 & 21.00 & 3.80 & 2.67 & 0.006 & 0.27 & 0.00 & 0.00 & 97.60 & 366.00 & 24.99 & 49.98 & 5.89 & 0.09 \\
\hline L19 & 27.20 & 17.67 & 2.00 & 1.33 & 0.035 & 0.14 & 24.00 & 0.00 & 146.40 & 268.40 & 34.99 & 44.99 & 6.24 & 0.00 \\
\hline L20 & 60.00 & 41.33 & 3.00 & 1.67 & 1.231 & 0.03 & 0.00 & 0.00 & 244.00 & 427.00 & 74.98 & 129.96 & 27.28 & 22.38 \\
\hline L21 & 68.00 & 42.67 & 13.60 & 3.00 & 0.265 & 0.02 & 0.00 & 0.00 & 475.80 & 915.00 & 154.95 & 194.94 & 10.57 & 3.20 \\
\hline L22 & 54.00 & 42.33 & 10.00 & 4.33 & 0.012 & 0.10 & 48.00 & 0.00 & 219.60 & 854.00 & 34.99 & 59.98 & 18.53 & 4.71 \\
\hline L23 & 6.60 & 2.33 & 3.60 & 2.67 & 0.023 & 0.02 & 0.00 & 0.00 & 97.60 & 231.80 & 24.99 & 15.00 & 1.56 & 1.24 \\
\hline L24 & 42.00 & 103.33 & 2.40 & 1.67 & 0.023 & 0.03 & 24.00 & 0.00 & 195.20 & 536.80 & 134.96 & 459.86 & 32.13 & 58.80 \\
\hline L25 & 13.20 & 5.67 & 2.80 & 1.67 & 3.577 & 0.05 & 0.00 & 0.00 & 48.80 & 85.40 & 19.99 & 19.99 & 9.09 & 0.00 \\
\hline L26 & 54.00 & 37.67 & 3.00 & 2.00 & 0.035 & 0.04 & 48.00 & 24.00 & 341.60 & 1000.40 & 19.99 & 24.99 & 4.16 & 0.00 \\
\hline L27 & 64.00 & 39.00 & 4.40 & 3.67 & 0.017 & 0.02 & 48.00 & 0.00 & 122.00 & 524.60 & 49.98 & 79.98 & 23.21 & 17.41 \\
\hline L28 & 33.80 & 32.00 & 3.40 & 2.33 & 0.069 & 0.12 & 24.00 & 0.00 & 170.80 & 573.40 & 69.98 & 89.65 & 8.23 & 3.11 \\
\hline L29 & 78.00 & 48.67 & 1.40 & 0.67 & 0.023 & 0.12 & 0.00 & 0.00 & 146.40 & 292.80 & 184.94 & 364.89 & 94.05 & 99.03 \\
\hline L30 & 66.00 & 47.00 & 3.20 & 2.00 & 0.012 & 0.50 & 0.00 & 0.00 & 244.00 & 427.00 & 129.96 & 87.34 & 50.14 & 43.97 \\
\hline L31 & 25.40 & 15.00 & 1.20 & 1.00 & 0.035 & 0.08 & 0.00 & 0.00 & 170.80 & 97.60 & 29.99 & 24.86 & 14.12 & 0.00 \\
\hline L32 & 23.80 & 23.67 & 3.00 & 2.00 & 0.035 & 0.46 & 24.00 & 24.00 & 244.00 & 646.60 & 19.99 & 39.99 & 4.33 & 2.66 \\
\hline L33 & 17.40 & 11.00 & 3.40 & 2.67 & 0.006 & 0.47 & 24.00 & 0.00 & 97.60 & 378.20 & 59.98 & 89.97 & 5.37 & 0.00 \\
\hline L34 & 27.00 & 20.33 & 1.60 & 1.00 & 0.017 & 0.20 & 48.00 & 24.00 & 146.40 & 524.60 & 44.99 & 154.95 & 9.79 & 6.31 \\
\hline L35 & 26.40 & 21.33 & 2.40 & 1.67 & 0.006 & 0.28 & 72.00 & 48.00 & 73.20 & 597.80 & 29.99 & 24.99 & 5.63 & 1.42 \\
\hline L36 & 30.20 & 28.33 & 3.00 & 1.67 & 0.046 & 0.12 & 24.00 & 0.00 & 195.20 & 512.40 & 24.99 & 44.99 & 11.26 & 5.60 \\
\hline Min. & 6.6 & 2.33 & 1.2 & 0.67 & 0 & 0.0 & 0.0 & 0.0 & 48.80 & 85.40 & 15.0 & 5.6 & 1.13 & 0.0 \\
\hline Max. & 102.0 & 103.33 & 13.6 & 14.00 & 3.577 & 2.53 & 72.0 & 144.0 & 475.80 & 1000.40 & 184.94 & 459.86 & 94.05 & 99.03 \\
\hline Mean & 41.57 & 35.83 & 4.01 & 2.45 & 0.175 & 0.21 & 24.75 & 10.33 & 175.21 & 460.89 & 60.26 & 96.57 & 19.22 & 15.35 \\
\hline Median & 34.90 & $34 / 8$ & 3.0 & 2.00 & 0.026 & 0.09 & 24.00 & 0.0 & 170.80 & 475.80 & 47.48 & 67.48 & 10.92 & 3.47 \\
\hline Std.Dev. & 22.37 & 22.14 & 2.80 & 2.24 & 0.618 & 0.42 & 23.91 & 26.93 & 83.82 & 216.07 & 41.37 & 96.14 & 19.57 & 22.46 \\
\hline
\end{tabular}

$T D S$ total dissolved solids (mg/l), EC electrical conductivity $(\mu \mathrm{S} / \mathrm{cm}), T A$ total alkalinity $(\mathrm{mg} / \mathrm{l}), T H$ total hardness (mg/l), $C a \mathrm{calcium}(\mathrm{mg} / \mathrm{l}), M g$ magnesium (mg/l), $\mathrm{Na}$ sodium (mg/l), $\mathrm{K}$ potassium (mg/l), $\mathrm{Fe}$ iron $(\mathrm{mg} / \mathrm{l}), \mathrm{CO}_{3}^{2-}$ carbonate $(\mathrm{mg} / \mathrm{l}), \mathrm{HCO}_{3}^{-}$bi-carbonate $(\mathrm{mg} / \mathrm{l}), \mathrm{Cl}^{-}$chloride $(\mathrm{mg} /$ 1), $\mathrm{SO}_{4}^{2-}$ sulfate $(\mathrm{mg} / \mathrm{l})$

The acceptable limit of magnesium in drinking water is considered as $30 \mathrm{mg} / \mathrm{l}$, though $100 \mathrm{mg} / \mathrm{l}$ is also used in case of no other alternative source (BIS 2012). Magnesium helps in maintaining normal nerve and muscle function, a healthy immune system and helps bones remain strong. It also helps in regulating glucose levels in blood and aids in the production of energy and protein. Deficiency of magnesium in the human diet might lead to anxiety, fatigue or anorexia. The magnesium concentration ranges between 1.56 and $115.36 \mathrm{mg} / \mathrm{l}$ with an average value of 31.21 in pre-monsoon 
Table 2 Values of calculated water quality parameters/indices

\begin{tabular}{|c|c|c|c|c|c|c|c|c|c|c|c|c|c|}
\hline \multirow{2}{*}{$\begin{array}{l}\text { Location } \\
\text { No. }\end{array}$} & \multirow[t]{2}{*}{ Location Name } & \multicolumn{2}{|l|}{ SAR } & \multicolumn{2}{|l|}{ SSP } & \multicolumn{2}{|l|}{ P.I } & \multicolumn{2}{|l|}{$\mathrm{RSC}$} & \multicolumn{2}{|l|}{ MAR } & \multicolumn{2}{|l|}{ KR } \\
\hline & & Pre & Post & Pre & Post & Pre & Post & Pre & Post & Pre & Post & Pre & Post \\
\hline L01 & Gosaidihi & 1.00 & 0.81 & 28.15 & 25.11 & 53.29 & 73.29 & -1.1395 & 1.975 & 33.55 & 42.07 & 0.38 & 0.30 \\
\hline L02 & Madhuban & 1.24 & 1.23 & 28.42 & 26.13 & 49.53 & 58.55 & -1.16617 & 1.3525 & 54.47 & 46.22 & 0.39 & 0.35 \\
\hline L03 & Gouripur & 0.97 & 0.67 & 23.89 & 14.13 & 51.57 & 27.15 & 0.050833 & -6.8975 & 34.75 & 68.61 & 0.30 & 0.16 \\
\hline L04 & Gajarbari & 0.42 & 0.16 & 23.54 & 5.39 & 88.35 & 60.10 & -0.08467 & 3.126667 & 21.53 & 91.38 & 0.26 & 0.05 \\
\hline L05 & Bagatpol & 1.09 & 0.93 & 33.56 & 28.05 & 70.76 & 67.52 & 0.688667 & 4.781667 & 65.37 & 34.75 & 0.45 & 0.37 \\
\hline L06 & Karanjora & 1.05 & 0.84 & 19.47 & 15.27 & 36.60 & 40.08 & -4.45183 & -0.26417 & 79.94 & 34.75 & 0.23 & 0.18 \\
\hline L07 & Mankanali & 1.62 & 2.66 & 34.41 & 54.10 & 59.11 & 104.81 & 0.411167 & 6.574167 & 80.57 & 60.01 & 0.50 & 1.16 \\
\hline L08 & Mujrakundi & 0.68 & 0.48 & 24.54 & 15.96 & 69.49 & 74.77 & -0.16933 & 2.7675 & 21.53 & 57.17 & 0.30 & 0.18 \\
\hline L09 & Khejurbedia & 0.98 & 0.94 & 25.49 & 24.79 & 46.29 & 58.55 & -1.09117 & 2.1525 & 57.17 & 47.91 & 0.29 & 0.27 \\
\hline L10 & Kanchanpur & 0.64 & 0.51 & 19.69 & 15.68 & 43.19 & 53.17 & -2.54367 & -0.4275 & 85.21 & 38.34 & 0.21 & 0.17 \\
\hline L11 & Tilabedya & 1.31 & 1.20 & 28.08 & 24.91 & 42.78 & 53.91 & -2.84517 & 0.553333 & 42.65 & 40.40 & 0.36 & 0.32 \\
\hline L12 & Beldangra & 1.94 & 1.37 & 38.34 & 26.76 & 59.17 & 48.74 & -1.18317 & -2.43917 & 74.07 & 33.28 & 0.60 & 0.36 \\
\hline L13 & Keshra & 0.94 & 0.72 & 30.71 & 23.99 & 64.06 & 80.25 & -0.975 & 1.574167 & 34.75 & 55.41 & 0.41 & 0.30 \\
\hline L14 & Sanabandh & 1.01 & 1.00 & 27.37 & 23.91 & 62.63 & 64.74 & -0.2645 & 3.145 & 39.14 & 64.03 & 0.36 & 0.31 \\
\hline L15 & Kanudihi & 1.58 & 2.16 & 27.10 & 37.10 & 40.71 & 65.34 & -3.726 & 5.16 & 79.27 & 34.37 & 0.36 & 0.58 \\
\hline L16 & Junbedia & 2.47 & 1.26 & 41.99 & 29.85 & 57.09 & 78.92 & -2.46783 & 6.761667 & 63.63 & 47.92 & 0.69 & 0.40 \\
\hline L17 & Bhadul & 1.33 & 0.99 & 31.37 & 22.41 & 49.98 & 61.44 & -0.53233 & 3.7525 & 70.35 & 46.22 & 0.44 & 0.28 \\
\hline L18 & Bikna & 0.87 & 0.80 & 26.19 & 27.26 & 51.63 & 95.20 & -1.93383 & 3.380833 & 23.94 & 43.88 & 0.33 & 0.35 \\
\hline L19 & Chhatarkanali & 1.04 & 0.77 & 32.45 & 28.54 & 72.82 & 103.18 & 0.630667 & 2.390833 & 21.53 & 26.84 & 0.46 & 0.38 \\
\hline L20 & Makurgram & 1.68 & 1.11 & 35.82 & 26.05 & 62.10 & 63.28 & -0.81283 & 1.776667 & 16.22 & 27.63 & 0.54 & 0.34 \\
\hline L21 & Madla & 1.18 & 0.86 & 20.73 & 17.25 & 36.87 & 51.51 & -4.83733 & 5.734167 & 76.07 & 43.34 & 0.23 & 0.20 \\
\hline L22 & Dhumura & 1.56 & 1.11 & 36.52 & 26.35 & 61.75 & 76.52 & 0.673333 & 8.545 & 62.89 & 61.50 & 0.52 & 0.34 \\
\hline $\mathrm{L} 23$ & Khemua & 0.32 & 0.09 & 19.07 & 6.542 & 81.81 & 81.15 & -0.00967 & 1.374167 & 37.39 & 65.37 & 0.18 & 0.04 \\
\hline L24 & Pratappur & 0.87 & 1.66 & 17.83 & 23.61 & 34.34 & 38.92 & -4.7 & -5.87167 & 42.07 & 29.86 & 0.21 & 0.31 \\
\hline L25 & Gopinathpur & 0.82 & 0.35 & 39.95 & 22.26 & 95.08 & 113.79 & -0.17017 & 0.39 & 65.37 & 58.42 & 0.59 & 0.24 \\
\hline L26 & Rasunkur & 1.56 & 0.99 & 34.85 & 23.66 & 68.52 & 80.26 & 2.667667 & 11.75167 & 70.35 & 53.75 & 0.52 & 0.30 \\
\hline $\mathrm{L} 27$ & Agaya & 1.85 & 1.09 & 39.07 & 27.05 & 57.51 & 70.95 & -0.91533 & 3.7725 & 47.91 & 34.75 & 0.62 & 0.35 \\
\hline L28 & Sonamela & 1.05 & 0.76 & 28.62 & 17.94 & 58.73 & 55.52 & -0.2815 & 2.763333 & 65.37 & 33.55 & 0.38 & 0.21 \\
\hline L29 & Helna-Susunia & 1.45 & 0.81 & 23.95 & 13.52 & 34.61 & 27.33 & -8.482 & -8.84083 & 1.196 & 18.41 & 0.31 & 0.16 \\
\hline L30 & Chelebakra & 1.35 & 0.97 & 24.69 & 19.16 & 41.02 & 43.09 & -5.00317 & -1.83833 & 29.09 & 26.34 & 0.32 & 0.23 \\
\hline L31 & Chendua & 1.04 & 1.18 & 33.54 & 52.77 & 82.82 & 152.29 & 0.550833 & 0.993333 & 25.31 & 65.38 & 0.49 & 1.08 \\
\hline L32 & Nekragaria & 0.71 & 0.69 & 20.97 & 19.59 & 58.08 & 78.42 & 0.609667 & 6.965 & 43.87 & 47.91 & 0.25 & 0.23 \\
\hline L33 & Basulitora & 0.52 & 0.31 & 16.80 & 10.17 & 40.96 & 55.94 & -1.779 & 1.3725 & 27.64 & 34.75 & 0.18 & 0.10 \\
\hline L34 & Kasibedia & 0.73 & 0.49 & 19.06 & 12.37 & 42.99 & 52.09 & -1.1605 & 2.956667 & 47.91 & 41.33 & 0.23 & 0.1 \\
\hline L35 & Muniadih & 0.76 & 0.65 & 21.12 & 19.44 & 39.61 & 82.01 & -0.91533 & 7.379167 & 47.91 & 32.10 & 0.25 & 0.23 \\
\hline L36 & Aralbanshi & 0.84 & 0.89 & 22.25 & 25.01 & 50.27 & 81.73 & -0.85733 & 4.578333 & 72.33 & 34.06 & 0.27 & 0.32 \\
\hline Min. & & 0.32 & 0.09 & 16.8 & 5.39 & 34.34 & 27.15 & -8.48 & -8.84 & 1.2 & 18.41 & 0.18 & 0.04 \\
\hline Max. & & 2.47 & 2.66 & 41.99 & 54.1 & 95.08 & 152.29 & 2.67 & 11.75 & 85.21 & 91.38 & 0.69 & 1.16 \\
\hline Mean & & 1.12 & 0.93 & 27.77 & 23.11 & 56 & 68.74 & -1.34 & 2.31 & 48.95 & 46.12 & 0.37 & 0.31 \\
\hline Median & & 1.04 & 0.875 & 27.23 & 23.78 & 55.19 & 65.04 & -0.92 & 2.58 & 47.91 & 43.61 & 0.36 & 0.3 \\
\hline Std. Dvn. & & 0.45 & 0.49 & 6.95 & 10.06 & 15.49 & 24.65 & 2.14 & 4.12 & 22.02 & 15.69 & 0.14 & 0.22 \\
\hline
\end{tabular}

SAR sodium adsorption ratio, SSP soluble sodium percentage, P.I. permeability index, RSC residual sodium carbonate, MAR magnesium adsorption ratio, $K R$ Kelly's ratio

and 4.76-71.61 mg/l with an average value of $28.00 \mathrm{mg} / \mathrm{l}$ in post-monsoon. Sodium also helps in regulating blood pressure levels in the human body. The sodium concentration varies from 6.60 to $102.00 \mathrm{mg} / \mathrm{l}$ with an average of $41.57 \mathrm{mg} / \mathrm{l}$ in pre-monsoon and $2.33-103.33 \mathrm{mg} / \mathrm{l}$ with an average of $35.83 \mathrm{mg} / \mathrm{l}$ in post-monsoon periods. 
Acceptable limit of calcium in drinking water is $75 \mathrm{mg} / \mathrm{l}$ (200 mg/l in case of no other alternative source) (BIS 2012). Calcium ion is necessary for proper mineralization of bones and bone strength. Deficiency in intake of calcium leads to eventual demineralization of bones for complementing the inadequate amounts of calcium in the body. Here $\mathrm{Ca}$ ranges from 6.72 to $215.04 \mathrm{mg} / \mathrm{l}$ in pre-monsoon and $4.20-222.60 \mathrm{mg} / \mathrm{l}$ in post-monsoon.

The sulfate ion causes no particular harmful effects on soils or plants; however, it contributes to increase the salinity in the soil solution. $\mathrm{SO}_{4}$ ranges from 1.13 to $94.05 \mathrm{mg} / \mathrm{l}$ in pre-monsoon and $0.00-99.03 \mathrm{mg} / \mathrm{l}$ in postmonsoon. Acceptable limit of sulfate in drinking water is $200 \mathrm{mg} / 1$ (400 mg/l in case of no other alternative source) (BIS 2012). Excess sulfate consumption through water might lead to occurrence of diarrhea in humans. $\mathrm{Cl}$ ranges from 15.0 to $184.94 \mathrm{mg} / \mathrm{l}$ in pre-monsoon and $15.0-459.86 \mathrm{mg} / \mathrm{l}$ in post-monsoon. WHO has set standards of 200-500 mg/l for chloride in drinking water. Too much of chloride leads to bad taste in water and also chloride ion combines with the $\mathrm{Na}$ (that is being derived from the weathering of granitic terrains) and forms $\mathrm{NaCl}$, whose excess presence in water makes it saline and unfit for drinking and irrigation purposes. Increase in chloride levels in our body might lead to increase in blood pressure levels and rise in body fluids. Bicarbonate ion varies from 48.8 to $475.8 \mathrm{mg} / \mathrm{l}$ and 85.4 to $1000.4 \mathrm{mg} / \mathrm{l}$ in pre- and post-monsoon, respectively. No standard limits have been provided by the Bureau of Indian Standards for level of carbonate and bicarbonate in drinking water.

\section{Drinking water suitability}

Domestic water mainly used for drinking and cooking purposes should be free from toxic chemicals and pathogens. The presence of certain anions and cations within permissible limits in groundwater are essential for human body. Table 3 shows the classification of samples according to standards specified for different water quality parameters. It is observed that no sample exceeds the maximum permissible limits for TDS, $\mathrm{pH}, \mathrm{Cl}, \mathrm{SO}_{4}, \mathrm{Mg}$ and $\mathrm{Na}$ in either pre-monsoon or post-monsoon. $2.78 \%$ of the total number of samples exceeds the maximum permissible limits for $\mathrm{HCO}_{3}$ in pre-monsoon and $61.11 \%$ of the samples exceeds in post-monsoon. $2.78 \%$ of the total number of samples exceeds the maximum permissible limits for $\mathrm{Ca}$ in pre-monsoon and $5.56 \%$ of the samples exceeds in postmonsoon. $5.56 \%$ of the total number of samples exceeds the maximum permissible limits for $\mathrm{Fe}$ in pre-monsoon and $2.78 \%$ of the samples exceeds in post-monsoon. Hard water is a measure of $\mathrm{Ca}$ and $\mathrm{Mg}$ in groundwater, expressed in equivalent of calcium carbonate. Water hardness increases the chance of heart diseases (WHO 2008). Hardness of water (temporary and permanent) is due to the soap action in water by the precipitation of $\mathrm{Ca}$ and $\mathrm{Mg}$ salts. Temporary hardness is due to the presence of calcium carbonate and gets removed when boiling water. Permanent hardness is caused by the presence of $\mathrm{Ca}$ and $\mathrm{Mg}$ which gets removed by ion exchange processes. The total hardness in $\mathrm{mg} / \mathrm{L}$ is determined by the following equation (Todd 1980). $2.78 \%$ of samples exceed the maximum permissible limits for $\mathrm{TH}$ in pre-monsoon and $5.56 \%$ of the samples exceeds in post-monsoon. $50 \%$ of the samples exceed the maximum permissible limits for EC in pre-monsoon and all of the samples exceed so in post-monsoon. According to classification by (Swayer and McCarty 1967) the TH of the groundwater shows that $55 \%$ of the samples are hard water and $22 \%$ are very hard water in pre-monsoon. In post monsoon $44 \%$ of the samples are hard water and 36 are very hard water. It is to be noted that for most of the parameters $>90 \%$ of the total number of samples is suitable for drinking purposes, i.e., they are within the permissible limits prescribed by (WHO 2008).

\section{Piper's diagram}

The hydrochemical evolution of groundwater is determined by plotting the cations and anions in Piper trilinear diagram (Piper 1944). This diagram reveals similarities and differences among water samples (Todd 1980). It consists of two lower triangular fields and a central diamond-shaped field (Fig. 3). All the three fields have scales reading in $\%$ of meq/l. The data points are pointed in two triangles and projected on to the diamond grid. The water quality types can be quickly identified by the location of points in the different zones of the diamond-shaped field, as shown in Fig. 3.

The trilinear diagram was developed from over 36 groundwater samples collected from the study area Bankura block I and II, Bankura district. The diagram divides in four distinct groups, fresh, saline, sulfate and alkaline. Premonsoon points are represented by circle and post-monsoon points are represented by triangle. With the help of this Piper trilinear diagram the water quality types can be quickly identified. The diagram indicates that $\mathrm{Ca}-\mathrm{Mg}-\mathrm{HCO}_{3}$ is the major water type dominant in pre-monsoon and $\mathrm{Ca}-\mathrm{HCO}_{3}$ is the major water type dominant in post-monsoon.

\section{Water quality index}

Water quality index determination (Tiwari and Mishra 1985) for a set of groundwater samples is undertaken with a major objective-to determine the suitability of 
Table 3 Classification of samples according to standards specified for different water quality parameters

\begin{tabular}{|c|c|c|c|c|c|c|}
\hline \multirow[t]{2}{*}{ Parameters } & \multirow[t]{2}{*}{ Range } & \multirow[t]{2}{*}{ Class } & \multicolumn{2}{|l|}{ No. of samples } & \multicolumn{2}{|c|}{ Percentage of samples } \\
\hline & & & Pre-monsoon & Post-monsoon & Pre-monsoon & Post-monsoon \\
\hline \multirow[t]{5}{*}{ SAR } & $<20$ & Excellent & 36 & 36 & 100 & 100 \\
\hline & $20-40$ & Good & 0 & 0 & 0 & 0 \\
\hline & $40-60$ & Permissible & 0 & 0 & 0 & 0 \\
\hline & $60-80$ & Doubtful & 0 & 0 & 0 & 0 \\
\hline & $>80$ & Unsafe & 0 & 0 & 0 & 0 \\
\hline EC & $<250$ & Excellent & 4 & 9 & 11 & 25 \\
\hline \multirow[t]{4}{*}{ WHO (2008) } & $250-750$ & Good & 14 & 27 & 39 & 75 \\
\hline & $750-2000$ & Permissible & 18 & 0 & 50 & 0 \\
\hline & $2000-3000$ & Doubtful & 0 & 0 & 0 & 0 \\
\hline & $>3000$ & Unsuitable & 0 & 0 & 0 & 0 \\
\hline $\mathrm{TH}$ & $<75$ & Soft & 2 & 2 & 6 & 6 \\
\hline \multirow[t]{3}{*}{ (Sawyer and McCarty 1967) } & $75-150$ & Moderate & 6 & 5 & 17 & 14 \\
\hline & $150-300$ & Hard & 20 & 16 & 55 & 44 \\
\hline & $>300$ & Very Hard & 8 & 13 & 22 & 36 \\
\hline \multirow[t]{3}{*}{ RSC } & $<1.25$ & Safe & 35 & 11 & 97 & 31 \\
\hline & $1.25-2.50$ & Marginally suitable & 0 & 7 & 0 & 19 \\
\hline & $>2.50$ & Unsuitable & 1 & 18 & 3 & 50 \\
\hline \multirow[t]{2}{*}{ MAR } & $<50$ & Suitable & 20 & 25 & 56 & 70 \\
\hline & $>50$ & Unsuitable & 16 & 11 & 44 & 30 \\
\hline \multirow[t]{2}{*}{ SSP } & $<200$ & Suitable & 36 & 36 & 100 & 100 \\
\hline & $>200$ & Unsuitable & 0 & 0 & 0 & 0 \\
\hline \multirow[t]{2}{*}{ KR } & $<1.0$ & Suitable & 36 & 34 & 100 & 94 \\
\hline & $>1.0$ & Unsuitable & 0 & 2 & 0 & 6 \\
\hline \multirow[t]{3}{*}{ PI } & $<80$ & Good & 32 & 26 & 89 & 72 \\
\hline & 80-100 & Moderate & 4 & 6 & 11 & 17 \\
\hline & $100-120$ & Poor & 0 & 4 & 0 & 11 \\
\hline \multirow[t]{5}{*}{ WQI } & $0-25$ & Excellent & 29 & 17 & 80 & 47 \\
\hline & $26-50$ & Good & 3 & 8 & 8 & 22 \\
\hline & $51-75$ & Poor & 1 & 1 & 3 & 3 \\
\hline & $76-100$ & Very Poor & 1 & 4 & 3 & 11 \\
\hline & $>100$ & Unfit for Drinking & 2 & 6 & 6 & 17 \\
\hline
\end{tabular}

groundwater of the present study area with respect to drinking. This index calculation involves use of the measure of total alkalinity and total hardness and concentrations of major cations and anions present in groundwaterall of which are generated through quantitative chemical analysis, values of parameters like $\mathrm{pH}$ and TDS which are measured in situ and the threshold limits set for each of the parameters by WHO or BIS (in case of absence of guideline from WHO).

The calculation of the WQI value for each water sample is carried out using the steps elaborated below (Eqs 1-4). On completion of all calculations suitability of each sample is judged using the classification standards set for this study presented in Table 3.
$\mathrm{WQI}=$ Anti $\log \left[W_{n=1}^{n} \log _{10} q_{n}\right]$,

where $W$ is the weightage factor, $q$ is the quality rating.

$W_{n}=K / S_{n}$

where the proportionality constant,

$K=\left[1 /\left(\sum_{n=1}^{n} 1 / S_{i}\right)\right]$,

where $S_{n}$ and $S_{i}$ are the standard/permissible values of water quality parameters, proposed by WHO or ICMR.

Quality rating,

$q=\left\{\left(V_{\text {actual }}-V_{\text {ideal }}\right) /\left(V_{\text {standard }}-V_{\text {ideal }}\right) \times 100\right\}$, 
Fig. 3 Plots of groundwater samples (both pre- and postmonsoon) in Piper's Trilinear diagram

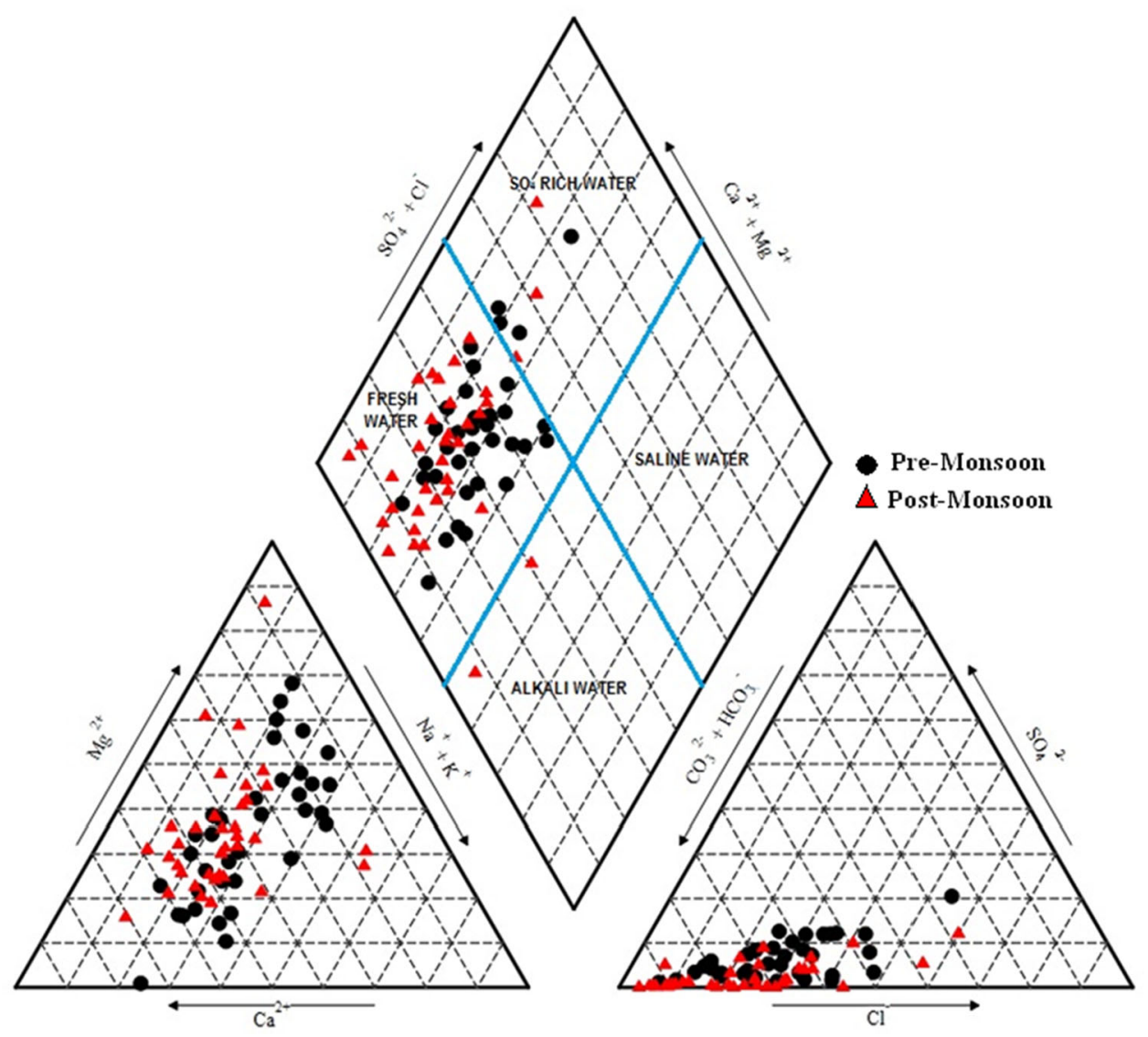

where $V_{\text {actual }}=$ Analytical value of $i$ th parameter obtained from laboratory analysis,

$V_{\text {standard }}=\mathrm{WHO} / \mathrm{ICMR}$ standard of $i$ th parameter,

$V_{\text {ideal }}=$ Value of $i$ th parameter obtained from standard tables $\left(V_{\text {ideal }}=0\right.$ for all parameters except $\mathrm{pH}$ where $V_{\text {ideal }}=7$ ).

According to the results of the WQI study carried out on groundwater sample data of the present study area it can be concluded that during the pre monsoon session combining the first two categories, i.e., excellent and good, $89 \%$ of the water samples are fit foe drinking purpose. A handful of samples out of 36 samples show spike in some ionic concentrations rendering them poor or unfit for drinking. During post monsoon though the results vary notably as calculations show that close to $30 \%$ of the samples are not suitable for drinking - which is a significant rise compared to pre monsoon trends, Table 3 and Fig. $4 a, b$.

\section{Irrigation suitability}

The quality of irrigation water depends mainly on six computed water quality parameters namely (1) soluble sodium percentage (SSP), (2) magnesium adsorption ratio
(MAR), (3) residual sodium carbonate (RSC), (4) sodium adsorption ratio (SAR), (5) permeability index (PI) and (6) Kelly's ratio (KR) (Ishaku 2011; Obiefuna and Shriff 2011). The above essential parameters of the present area for both pre-monsoon and post-monsoon are produced in Table 2. Electrical conductivity (EC) of the Groundwater samples was measured and is produced in Table 3. EC data indicates that in pre-monsoon the water lies between excellent and permissible and in post-monsoon the water lies between excellent and good.

\section{Soluble sodium percentage (SSP)}

Sodium is an important ion used for the classification of irrigation water due to its reaction with soil, which reduces its permeability. Sodium is usually expressed in terms of percent sodium or soluble-sodium percentage $(\% \mathrm{Na})$. Percentage of $\mathrm{Na}^{+}$is widely used for assessing the suitability of water for irrigation purposes (Wilcox 1955). The soluble sodium percentage (SSP), an important parameter of the groundwater has been calculated by using the formula (Raghunath 1987):

$\mathrm{SSP}=[(\mathrm{Na}+\mathrm{K}) /(\mathrm{Ca}+\mathrm{Mg}+\mathrm{Na}+\mathrm{K})] \times 100$. 


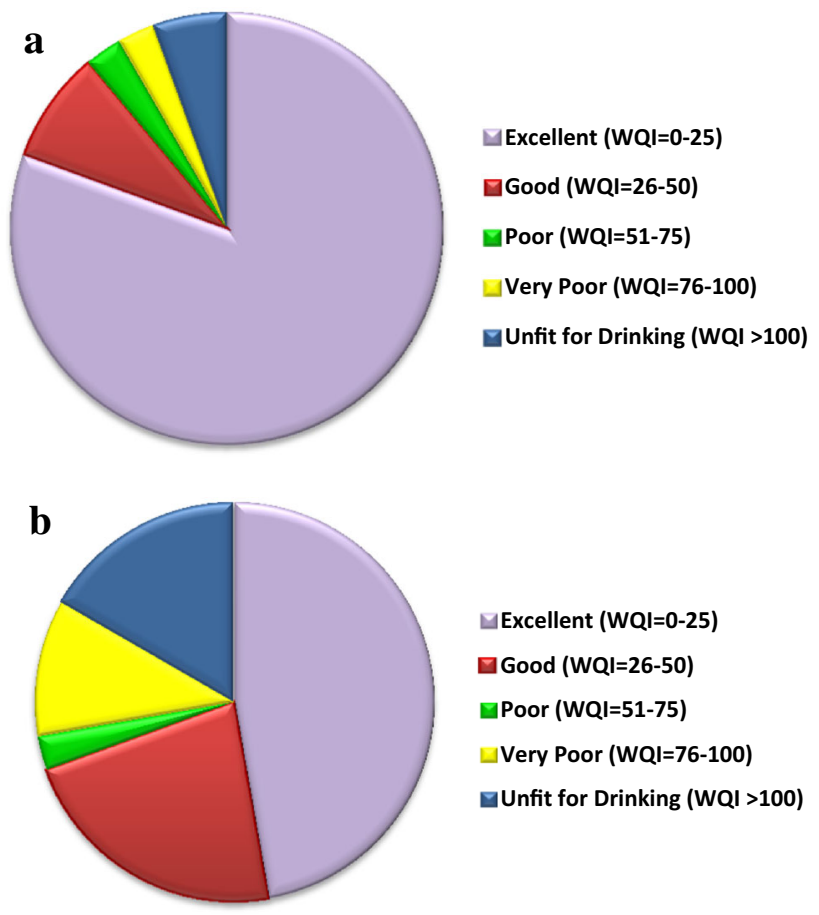

Fig. 4 a Pie-chart representing WQI results for pre-monsoon session. b Pie-chart representing WQI results for post-monsoon session

The calculated soluble sodium percentage is shown in Table 2. From the Table 3 it is observed that in both preand post-monsoon, all the samples are suitable for irrigation. In Fig. 5 (Wilcox 1955) the plot of SSP vs. $\mathrm{EC}$ of the groundwater samples in the area has been shown. This shows that all the samples lie within very good to permissible fields. So the groundwater is suitable for irrigation in the area throughout the year.

\section{Magnesium adsorption ratio (MAR)}

Generally $\mathrm{Ca}^{2+}$ and $\mathrm{Mg}^{2+}$ maintain a state of equilibrium in most groundwater (Hem 1985). During equilibrium more $\mathrm{Mg}^{2+}$ in groundwater will adversely affect the soil quality rendering it alkaline resulting in decrease of crop yield (Kumar et al. 2007). Paliwal (1972) developed an index for calculating the magnesium hazard (magnesium ratio (MR) where calcium and magnesium ratios are taken into consideration, as mostly calcium and magnesium maintain equilibrium in water (Giggenbach 1988). Magnesium adsorption ratio (MAR) for irrigation water of the present area has been calculated by using the formula:

MAR $=\left(\mathrm{Mg}^{2+} \times 100\right) /\left(\mathrm{Ca}^{2+}+\mathrm{Mg}^{2+}\right)$,

where all ions are calculated in meq/l.

Irrigation water with MAR above 50 is usually not suitable. In pre-monsoon $56 \%$ of the samples show MAR

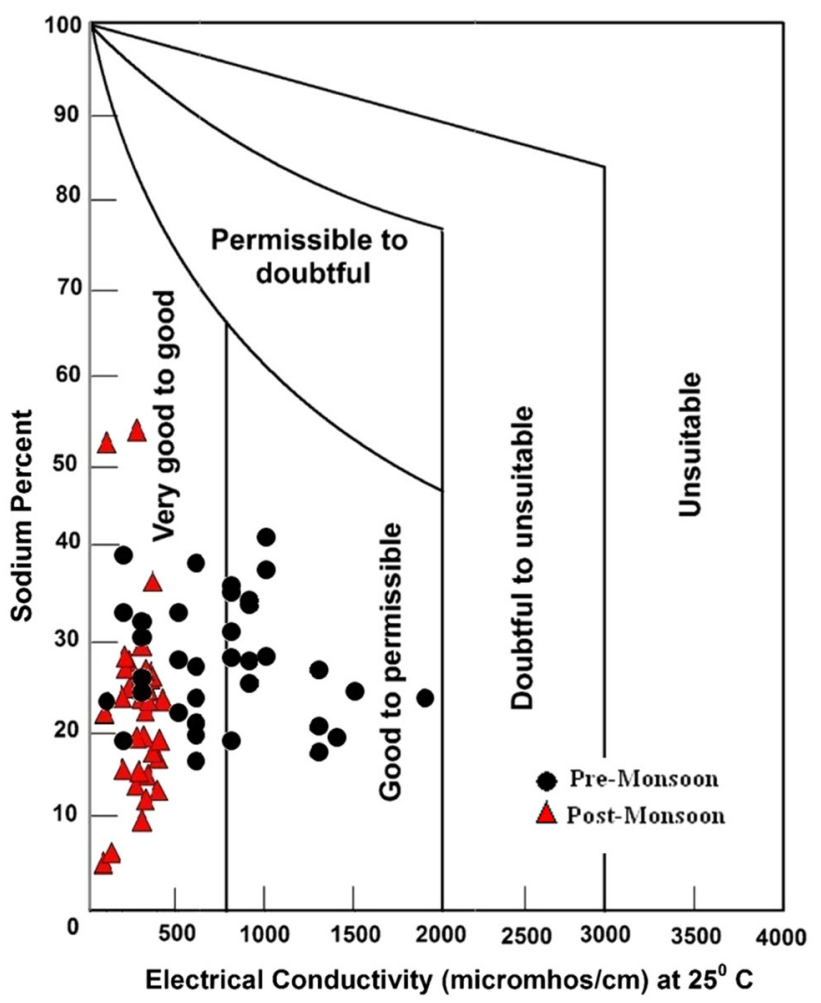

Fig. 5 Plots of groundwater samples (both pre- and post-monsoon) in Wilcox diagram

value less than 50 , whereas in post-monsoon $70 \%$ of the samples show MAR value less than 50. The samples having MAR values above the permissible limit of $50 \mathrm{mg} / \mathrm{l}$ indicating the unfavorable effect on crop yield and increase the soil alkalinity. Those samples would adversely affect the crop yield by making it more alkaline (Paliwal 1972).

\section{Residual sodium carbonate (RSC)}

Residual sodium carbonate (RSC) is calculated to determine the hazardous effect of carbonate and bicarbonate on the quality of water used for agricultural activities (Srinivasamoorthy et al. 2011b; Raju 2007). Suitability of groundwater used for irrigation depends upon the concentration of bicarbonate and carbonate higher than calcium and magnesium. Residual sodium carbonate (RSC) values of the water samples of the present area have been calculated using the formula (Raghunath 1987):

$\mathrm{RSC}=\left(\mathrm{HCO}_{3}^{-}+\mathrm{CO}_{3}^{2-}\right)-\left(\mathrm{Ca}^{2+}+\mathrm{Mg}^{2+}\right)$,

where all ions are calculated in meq/l.

A negative RSC value indicates that sodium build-up is unlikely since sufficient calcium and magnesium are in excess of that can be precipitated as carbonates. Whereas a positive RSC value indicates that sodium build-up in the 
Fig. 6 Plots of groundwater samples (both pre- and postmonsoon) in U. S. Salinity diagram

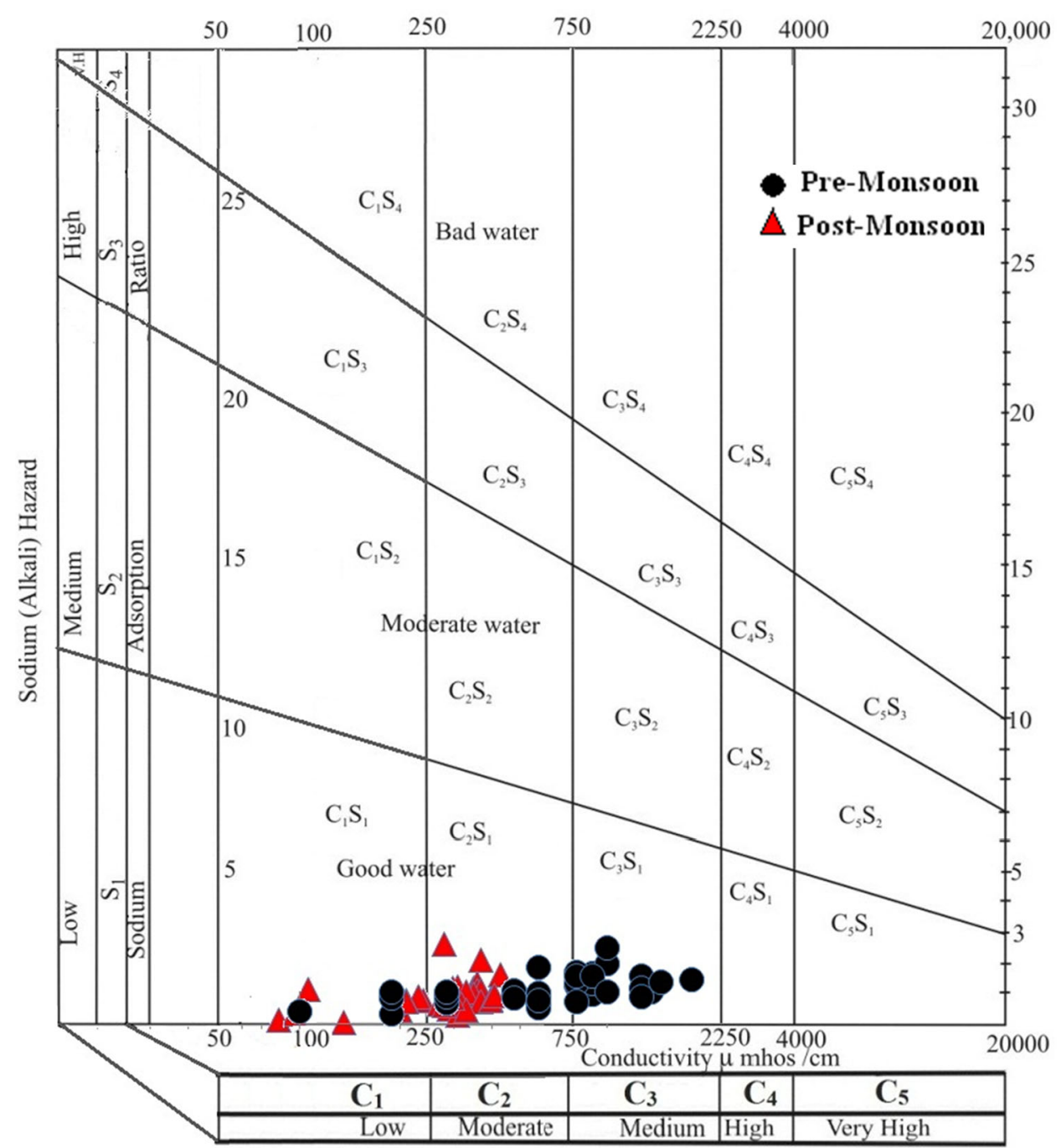

$\left.\mathrm{SAR}=\left[\mathrm{Na}^{+}\right] /\left\{\left[\mathrm{Ca}^{2+}+\mathrm{Mg}^{2+}\right]\right) / 2\right\}^{1 / 2}$,

soil is possible. It is observed that in the present area in premonsoon most of the groundwater samples are within safe zone but in post-monsoon $50 \%$ of the groundwater samples fall in unsuitable zone.

\section{Sodium adsorption ratio (SAR)}

Total salt concentration and probable sodium hazard of the irrigation water are the two major constituents for determining sodium adsorption ratio (SAR). Salinity hazard is based on EC measurements. If water used for irrigation is high in $\mathrm{Na}^{+}$and low in $\mathrm{Ca}^{2+}$ the ion exchange complex may become saturated with $\mathrm{Na}^{+}$which destroys the soil structure, due to the dispersion of clay particles (Todd 1980) and reduces the plant growth. Excess salinity reduces the osmotic activity of plants (Subramani et al. 2005). The sodium adsorption ratio (SAR) of the irrigation water is another important parameter and it is calculated according to Richards (1954) using the formula: where all ions are calculated in meq/l.

On the basis of SAR irrigation water is classified into four categories $\mathrm{C}_{1}, \mathrm{C}_{2}, \mathrm{C}_{3}$ and $\mathrm{C}_{4}$. The sodium hazard is classified into four groups $S_{1}, S_{2}, S_{3}$ and $S_{4}$. The obtained values are plotted in the US Salinity Laboratory (1954) diagram to find out suitability of irrigation water (Fig. 6). In the present area in pre-monsoon all groundwater samples lie within $C_{1} S_{1}$ and $\mathrm{C}_{2} \mathrm{~S}_{1}$ fields. In post-monsoon all the values lie in $\mathrm{C}_{1} \mathrm{~S}_{1}, \mathrm{C}_{2} \mathrm{~S}_{1}$ and $\mathrm{C}_{3} \mathrm{~S}_{1}$ fields. So it is evident that all the groundwater samples are suitable for irrigation purposes throughout the year according to US Salinity Diagram.

\section{Permeability index (PI)}

The permeability of soil is affected by long-term use of irrigation water and is influenced by sodium, calcium, magnesium and bicarbonate contents in soil. The 


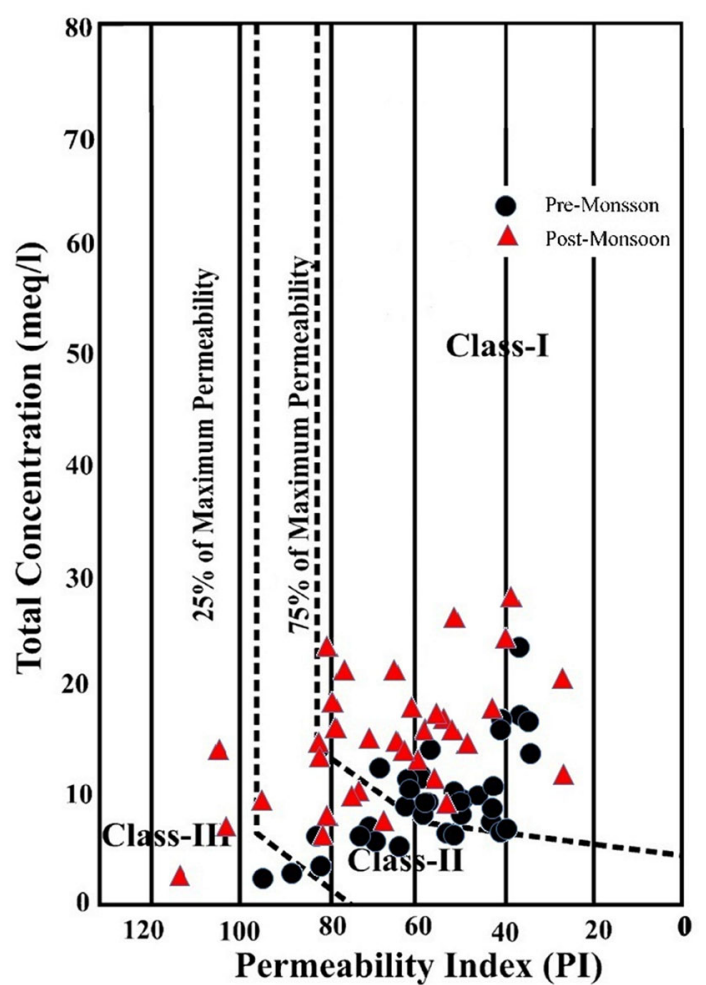

Fig. 7 Plots of groundwater samples (both pre- and post-monsoon) in Domeen's diagram

Permeability index (PI) of the groundwater samples have been determined after Doneen (1964), using the formula:

$$
\begin{aligned}
\mathrm{PI}= & \mathrm{Na}^{+} \\
& +\left[\left\{\left(\mathrm{HCO}_{3}^{-}\right)^{1 / 2} /\left(\mathrm{Ca}^{2+}+\mathrm{Mg}^{2+}+\mathrm{Na}^{+}\right)\right\} \times 100\right],
\end{aligned}
$$

where all ions are calculated in meq/l.

According to PI values, the groundwater samples falling in class I and class II indicate that the water is moderate to good for irrigation purposes (Arumugam and Elangovan 2009). Waters falling under Class III are not suitable for irrigation. In the present area (Fig. 7) $94 \%$ of the groundwater samples fall in Class I and Class II in pre-monsoon. In post monsoon $100 \%$ of the groundwater samples fall in good to moderate category in pre-monsoon time whereas $89 \%$ samples fall in good to moderate category in post-monsoon time. Thus, it is imperative to say that the overall quality of groundwater is suitable for irrigation throughout the year.

\section{Kelly's ratio (KR)}

Kelly's ratio (KR) is defined as the excess amount of sodium over calcium and magnesium. KR is used to find out the suitability of groundwater for irrigation. According to Kelly (1963), the KR is expressed by the equation:
$\mathrm{KR}=\mathrm{Na}^{2+} /\left(\mathrm{Ca}^{2+}+\mathrm{Mg}^{2+}\right)$,

where concentration of all constituents are expressed in meq/l.

In this study we observe that all samples are suitable for irrigation in pre-monsoon and $94 \%$ of samples are suitable for irrigation in post-monsoon.

\section{Gibb's diagrams}

The hydrogeochemistry of a particular region is usually determined by a number of factors like climate (average temperature of the region), geology (composition of the underlying bed rocks lining the aquifer systems in the region), rainfall, etc. Plotting of values of specific water quality parameters over the Gibb's diagram (Gibbs 1970) gives us an insight as to which particular factor-evaporation, precipitation or rock-water interaction, plays the dominant role in controlling the hydrogeochemistry of an area. Gibb's diagram is prepared using TDS, sodium $\left(\mathrm{Na}^{+}\right)$, potassium $\left(\mathrm{K}^{+}\right)$, calcium $\left(\mathrm{Ca}^{2+}\right)$, chloride $\left(\mathrm{Cl}^{-}\right)$and bicarbonate $\left(\mathrm{HCO}_{3}{ }^{-}\right)$concentrations in groundwater. In Fig. 8a, b the Gibbs's diagrams for pre monsoon and post monsoon sessions have been presented. From these diagrams it can be interpreted that during both sessions rockwater interaction processes significantly control the levels of all chemical constituents in groundwater of the study area. Dissolution and displacement reactions in rocks lining the aquifers are primary reasons behind changing concentrations of major ions in solution.

\section{Conclusion}

To find out the groundwater suitability for domestic and agricultural purposes in Bankura Block I and II, 36 samples of groundwater from different bore wells have been studied in detail for both pre-monsoon and post-monsoon 2012. Hydrochemical studies have been carried out in details for the collected samples. The groundwater quality of the two blocks reveals that $\mathrm{pH}$ and TDS values of groundwater were safe for drinking and irrigation purposes. Other elements such as iron $(\mathrm{Fe})$, calcium $(\mathrm{Ca})$, magnesium $(\mathrm{Mg})$, sodium $(\mathrm{Na})$, chloride $(\mathrm{Cl})$, bicarbonate $\left(\mathrm{HCO}_{3}^{-}\right)$, and sulfate $\left(\mathrm{SO}_{4}^{=}\right)$are within the permissible limits except at some places where higher concentrations are beyond allowable limits. SAR values are excellent in all the samples, so the water is suitable for irrigation purpose. From Piper's diagram, it can be stated that water samples of some areas of the block are fresh and some areas have sulfate-rich water throughout the year, they are suitable for drinking and irrigation purpose, respectively. SSP values of pre-

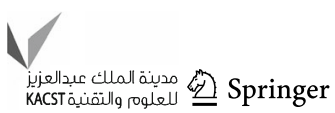



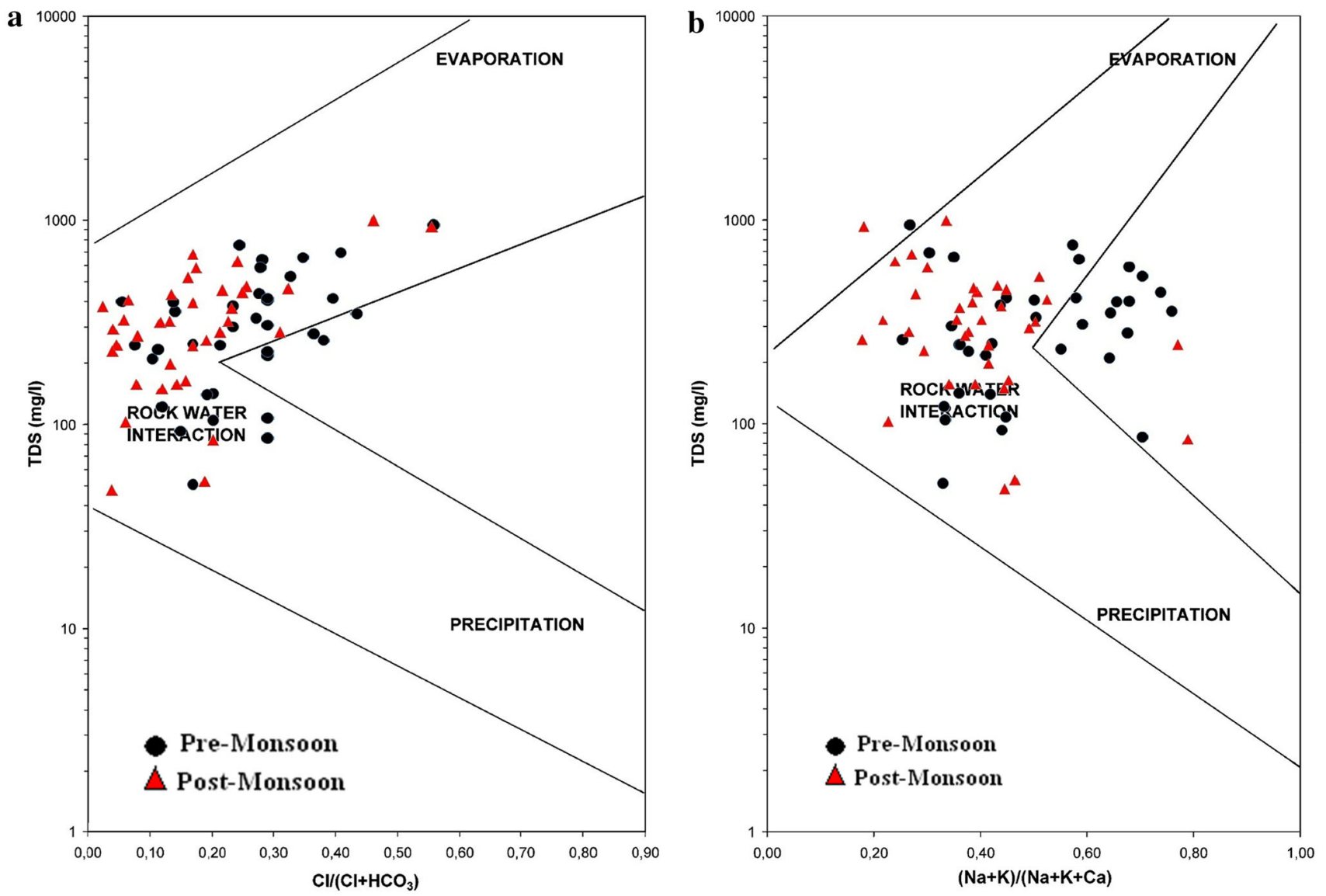

Fig. 8 a Gibb's Diagram for Post and Pre Monsoon Sampling Sessions. b Gibb's diagram for pre- and post-monsoon sampling sessions

monsoon and post-monsoon water samples also fall in very good to good zones and good to permissible categories.

The results from the water analysis have been used as a tool to identify the process and mechanisms affecting the chemistry of groundwater from the study area. The data points of the area are plotted on the Gibbs' (1970) diagram. The plot is used to determine the mechanism controlling the water chemistry (Fig. 8a, b). The samples fall in rockwater interaction dominant zone indicating chemical weathering of rock-forming minerals as the prime factor influencing the groundwater quality suggesting dissolution and displacement of minerals constituting the aquifer materials.

Hence, the study has helped to improve understanding of water quality of the area for effective management and proper utilization of groundwater resources for better living conditions of the people. A continuous monitoring program of water quality is required to avoid further deterioration of the water quality of the study area.

Acknowledgement The author (SKN) gratefully acknowledges University Grants Commission (UGC), Govt. of India for financial support through Major Research Project [F.No. 41-1045/2012 (SR)].
The author is also grateful to Dr. S. Gupta of Burdwan University for his support in analyzing the water quality parameters.

Open Access This article is distributed under the terms of the Creative Commons Attribution 4.0 International License (http:/ creativecommons.org/licenses/by/4.0/), which permits unrestricted use, distribution, and reproduction in any medium, provided you give appropriate credit to the original author(s) and the source, provide a link to the Creative Commons license, and indicate if changes were made.

\section{References}

Acworth RI (1987) The development of crystalline basement aquifers in a tropical environment. Q J Eng Geol 20:265-272

Aghazadeh N, Mogaddam AA (2010) Assessment of groundwater quality and its suitability for drinking and agricultural uses in the Oshnavieh area, Northwest of Iran. J Environ Prot 1:30-40

Ahmed AA (2007) Using lithologic modeling techniques for aquifer characterization and groundwater flow modeling of Sohag area, Egypt. Second International Conference on Geo-Resources in the Middle East and North Africa. 24-28 Feb. 2007, Cairo University, Egypt

Ahmed SS, Mazumder H, Jahan CS, Ahmed M, Islam S (2002) Hydrochemistry and classification of groundwater, Rajshahi City Corporation Area, Bangladesh. J Geol Soc Ind 60:411-418 
Al-Futaisi A, Rajmohan N, Al-Touqi S (2007) Groundwater quality monitoring in and around Barka dumping site, Sultanate of Oman. The Second IASTED (The International Association of Science and Technology for Development) International Conference on Water Resources Management (WRM 2007), Honolulu, Hawaii, USA, 20-22 August

APHA (Americal Public Health Association) (1998) Standard methods for the examination of water and wastewater, 20th edn. American Public Health Association, Washington DC

Arumugam K, Elangovan K (2009) Hydrochemical characteristics and groundwater quality assessment in Tirupur Region, Coimbatore District, Tamil Nadu, India. Environ Geol 58:1509-1520

Bathrellos GD, Skilodimou HD, Kelepertsis A, Alexakis D, Chrisanthaki I, Archonti D (2008) Environmental research of groundwater in the urban and suburban areas of Attica region, Greece. Environ Geol 56:11-18

BIS (Bureau of Indian Standards):10500 (2012) Indian standard drinking water specification, First revision 19911-19918

Brindha K, Elango L (2010) Study on bromide in groundwater in parts of Nalgonda district, Andhra Pradesh, India. Earth science India 3(1):73-80

Brindha K, Elango L (2011) Hydrochemical characteristics of groundwater for domestic and irrigation purposes in Madhuranthakam, Tamil Nadu, India. Earth Sci Res J 15(2):101-108

Brindha K, Rajesh R, Murugan R, Elango (2011) Fluoride contamination in groundwater in parts of Nalgonda district, Andhra Pradesh, India. Environ Monit Assess 172:481-492

Central Water Commission (CWC) (2006) Water and related statistics. Central Water Commission, Ministry of Water Resources, Government of India, New Delhi

Dart FJ (1974) The hazard of iron. Water and Pollution Control, Ottawa

Dewandel B, Lachassagne P, Wyns R, Maréchal JC, Krishnamurthy NS (2006) A generalized 3-D geological and hydrogeological conceptual model of granite aquifer controlled by single or multiphase weathering. J Hydrol 330:260-284

Domenico PA (1972) Concepts and models in groundwater hydrology. McGraw-Hill, New York

Doneen LD (1964) Water quality for agriculture. Department of irrigation, University of California, Davis, p 48

Freeze RA, Cherry JA (1979) Groundwater. Prentice Hall Inc, Englewood cliffs, p 604

Gibbs RJ (1970) Mechanisms controlling world's water chemistry. Science 170:1088-1090

Giggenbach WF (1988) Geothermal solute equilibria, derivation of $\mathrm{Na}-\mathrm{K}-\mathrm{Mg}-\mathrm{Ca}$ geoindicators. Geochim Cosmochim Acta 52(12):2749-2765

Hem JD (1985) Study and interpretation of the chemical characteristics of Natural water (3rd edn): U.S. Geological Survey, Water Supply Paper 2254

Hussein MT (2004) Hydrochemical evaluation of groundwater in the Blue Nile Basin, eastern Sudan, using conventional and multivariate techniques. Hydrogeol J 12:144-158

Ishaku JM (2011) Assessment of groundwater quality index for Jimeta-Yola area, northeastern Nigeria. J Geol Mining Res 3(9):219-231

Jalali M (2007) Hydrochemical identification of groundwater resources and their changes under the impacts of human activity in the Chah basin in western Iran. Environ Monit Assess 130:347-364

Kelly WP (1963) Use of saline irrigation water. Soil Sci 95(4):355-391

Krishnamurthy NS, Chandra S, Dewashisk K (2008) Geophysical characterisation of hard rock aquifers. In: Ahmed S, Jayakumar S, Salih A (eds) Groundwater dynamics in hard rock aquifers. Springer, Netherlands, p 64-86
Kumar R, Singh RD, Sharma KD (2005) Water resources India. Curr Sci 89:794-811

Kumar M, Kumari K, Ramanathan AL, Saxena R (2007) A comparative evaluation of groundwater suitability for irrigation and drinking purposes in two intensively cultivated districts of Punjab, India. Environ Geol 53:553-574

Lloyd JW (1999) Water resources of hard rock aquifers in arid and semi-arid zones. Studies and Reports in Hydrology, 58, UNESCO, Paris

Milovanovic M (2007) Water quality assessment and determination of pollution sources along the Axios/Vardar River, Southeast Europe. Desalination 213:159-173

Mohan R, Singh AK, Tripathi JK, Chowdhary GC (2000) Hydrochemistry and quality assessment of ground water in Naini Industrial area, Allahabad district, Uttar Pradesh. J Geol Soc Ind 55:77-89

Moore CV (1973) Iron in: modern nutrition in health and disease. Philadelphia, Lea and Febiger, p 297

Mukherjee S, Das AK (2007) Groundwater quality assessment for irrigation and domestic uses in Raigad district, Maharashtra, India. J Earth Sci 1(1):66-81

Nag SK (2014) Evaluation of hydrochemical parameters and quality assessment of the groundwater in Gangajalghati Block, Bankura District, West Bengal, India. Arab J Sci Eng 39:5715-5727

Nag SK, Ghosh P (2013) Variation in groundwater levels and water quality in Chhatna Block, Bankura District, West Bengal-A GIS approach. J Geol Soc India 81(2):261-280

Nag SK, Ray S (2015) Hydrochemical evaluation of groundwater quality of Bankura I and II Blocks, Bankura District, West Bengal, India: emphasis on irrigation and domestic utility. Arab J Sci Eng 40:205-214

Obiefuna GI, Sheriff A (2011) Assessment of Shallow ground water quality of Pindiga Gombe Area, Yola Area, NE, Nigeria for irrigation and domestic purposes. Res J Environ Earth Sci 3(2):131-141

Paliwal KV (1972) Irrigation with saline water. Monogram no. 2 (New series). New Delhi, IARI, 198

Piper AM (1944) A graphical procedure in the geochemical interpretation of water analysis. Trans Am Geophys Union 25:914-928

Pritchard M, Mkandawire T, O'Neill JG (2008) Assessment of groundwater quality in shallow wells within the southern districts of Malawi. Phys Chem Earth 33:812-823

Raghunath HM (1987) Ground Water 2nd Edn, Wiley Eastern Limited

Rajmohan N, Al-Futaisi A, Jamrah A (2007) Evaluation of long-term groundwater level data in regular monitoring wells, Barka, Sultanate of Oman. Hydrol Process 2z:3367-3379

Raju NJ (2007) Hydrogeochemical parameters for assessment of groundwater quality in the upper Gunjanaeru River basin, Cuddapah District, Andhra Pradesh, South India. Environ Geol 52:1067-1074

Ramesh K, Elango L (2005) Groundwater quality assessment in Tondiar basin. Indian J Environ Prot 26(6):497-504

Richards LA (1954) Diagnosis and improvement of saline and alkali soils. US department of agriculture, Handbook, p 60

Rivers CN, Hiscock KM, Feast NA, Barrett MH, Dennis PF (1996) Use of nitrogen isotopes to identify nitrogen contamination of the Sherwood sandstone aquifer beneath the city of Nottingham. UK. Hydrol J 4(1):90-102

Schuh WM, Klinekebiel DL, Gardner JC, Meyar RF (1997) Tracer and nitrate movements to groundwater in the Norruem Great Plains. J Environ Qual 26:1335-1347

Sharma HD, Chawla AS (1977) Manual on ground water and tube wells. Technical report 18. Central Board of Irrigation and Power

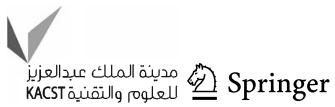


Singhal BBS, Gupta RP (2010) Applied hydrogeology of fractured rocks, 2nd edn. Springer, New York

Sreedevi PD (2004) Groundwater quality of Pageru river basin, Cuddapah district, Andhra Pradesh. J Geol Sci India 64:619-636

Srinivasamoorthy K, Chidambaram S, Vasanthavigar M (2008) Geochemistry of fluorides in groundwater: salem District, Tamil Nadu, India. J Environ Hydrol 1:16-25

Srinivasamoorthy K, Nanthakumar C, Vasanthavigar M (2011) Groundwater quality assessment from a hard rock terrain, Salem district of Tamilnadu, India. Arab J Geosci 4(1):91-102

Srinivasamoothy K, Vijayaraghavan Kannusamy, Murugesan Vasanthavigar, Rajivgandhi R, Sarma VS (2011) Integrated techniques to identify groundwater vulnerability to pollution in a highly industrialized terrain. Environ Monit Assess, Tamil Nadu. doi:10.1007/s10661-010-1857-x

Stites W, Kraft GJ (2001) Nitrate and chloride loading to groundwater from an irrigated North-Central U.S, Sand-plain vegetable field. J Environ Qual 30:1176-1184

Subba Rao N, Prakasa Rao J, John Devadas D, Srinivasa Rao KV, Krishna C, Nagamalleswara Rao B (2002) Hydrogeochemistry and groundwater quality in a developing urban environment of a semi-arid region, Guntur, Andhra Pradesh. J Geol Soc Ind 59:159-166

Subramani T, Elango L, Damodarasamy SR (2005) Groundwater quality and its suitability for drinking and agricultural use in Chithar River Basin, Tamil Nadu, India. Environ Geol 47:1099-1110

Swayer GN, McCarty DL (1967) Chemistry of sanitary engineers, 2nd edn. McGraw hill, New York, p 518
Tiwari TN, Mishra M (1985) A preliminary assignment of water quality index of major Indian rivers. Indian J Environ Prot 5(4):276-279

Todd DK (1980) Ground water hydrogeology. Wiley, New York

UNESCO (2007) UNESCO water portal newsletter no. 161. Water related diseases. http://www.unesco.org/ water/news/newsletter/ 161.shtml

USSL (United States Salinity Laboratory) (1954) Diagnosis and improvement of saline and alkali soils. USDA Handbook 60:147

Vasanthavigar M, Srinivasamoorthy K, Vijayaragavan K, Rajiv Ganthi R, Chidambaram S, Sarama VS, Anandhan P, Manivannan R, Vasudevan S (2010) Application of water quality index for groundwater quality assessment: Thirumanimuttar SubBasin, Tamilnadu, India. Environ Monit Assess 171(1-4):595-609

Walton WC (1970) Groundwater resources evaluation. McGraw Hill Book Co, New York

WHO (2008) Guidelines for drinking water quality, 3rd edn. World Health Organization, Geneva

WHO/UNICEF (2004) Meeting the MGD drinking water and sanitation target: a mid-term assessment of progress. WHO, Geneva

Wilcox LV (1955) Classification and use of irrigation waters. US department of agriculture. Circular 969. Washington DC, USA

Wright EP, Burgess WG (eds) (1992) The hydrogeology of crystalline basement aquifers in Africa. Soc Spl Publ N, Geol 